\title{
Far infrared and radio emission in dusty starburst galaxies
}

\author{
A. Bressan ${ }^{1,2}$, L. Silva ${ }^{3}$, and G. L. Granato ${ }^{1,2}$ \\ 1 INAF, Osservatorio Astronomico di Padova, Vicolo Osservatorio 5, 35122 Padova, Italy \\ 2 SISSA, Strada Costiera, 34131 Trieste, Italy \\ 3 INAF, Osservatorio Astronomico di Trieste, Via Tiepolo 11, 34131 Trieste, Italy
}

Received 11 January 2002 / Accepted 31 May 2002

\begin{abstract}
We revisit the nature of the far infrared (FIR)/radio correlation by means of the most recent models of star forming galaxies, focusing in particular on the case of obscured starbursts. We model the IR emission with our population synthesis code, GRASIL (Silva et al. 1998). For the radio emission, we revisit the simple model of Condon \& Yin (1990). We find that a tight FIR/radio correlation is natural when the synchrotron mechanism dominates over the inverse Compton, and the electron cooling time is shorter than the fading time of the supernova $(\mathrm{SN})$ rate. Observations indicate that both these conditions are met in star forming galaxies, from normal spirals to obscured starbursts. However, since the radio non-thermal (NT) emission is delayed, deviations are expected both in the early phases of a starburst, when the radio thermal component dominates, and in the post-starburst phase, when the bulk of the NT component originates from less massive stars. We show that this delay allows the analysis of obscured starbursts with a time resolution of a few tens of Myrs, unreachable with other star formation (SF) indicators. We suggest a strategy to complement the analysis of the deviations from the FIR/radio correlation with the radio slope ( $q$-radio slope diagram) to obtain characteristic parameters of the burst, e.g. its intensity, age and fading time scale. The analysis of a sample of compact ULIRGs shows that they are intense but transient starbursts, to which one should not apply usual SF indicators devised for constant SF rates. We also discuss the possibility of using the $q$-radio slope diagram to assess the presence of obscured AGN. A firm prediction of the models is an apparent radio excess during the post-starburst phase, which seems to be typical of a class of star forming galaxies in rich cluster cores. Finally we discuss how deviations from the correlation, due to the evolutionary status of the starburst, affect the technique of photometric redshift determination widely used for high- $z$ sources.
\end{abstract}

Key words. ISM: dust, extinction - galaxies: stellar content - infrared: galaxies - radio continuum: galaxies

\section{Introduction}

In recent years the study of starburst galaxies has become a very popular subject because of its intimate connection with the global star formation history of the Universe. On one side high redshift observations in the optical bands probe rest frame spectral regions that are highly affected by even tiny amounts of ongoing star formation and dust extinction. On the other, theoretical models following the paradigm of the hierarchical clustering scenario predict that merging induced star formation should have been highly enhanced in the past. Current estimates of the star formation rate (SFR) of the Universe have thus been interpreted on the basis of our understanding of local analogous galaxies, in particular through UV continuum and optical line emission. However, in local starbursts a significant fraction of the ongoing star formation may be hidden to UV and optical estimators. In fact, though starburst galaxies were initially selected for the prominence of their optical emission lines, it appears that this criterion excludes other actively

Send offprint requests to: A. Bressan,

e-mail: bressan@pd.astro.it star forming objects and possibly limits our understanding to a small phase of their evolution. After the IRAS satellite, it became clear that SF is also highly enhanced in very and ultra luminous infrared galaxies that are otherwise highly attenuated in the optical. With space densities similar to those of quasars (Soifer et al. 1986) and total infrared luminosities spanning the range $10^{11}-10^{12} L_{\odot}$ and above $10^{12} L_{\odot}$, respectively, Luminous and Ultraluminous Infrared galaxies (LIRGs and ULIRGs) are the most luminous objects in the local Universe. Evidence of the important role played by dust reprocessing was provided by the detection of a diffuse FIR background whose high intensity (equal to or higher than that of the optical, e.g. Hauser et al. 1998) implies that these galaxies are undergoing intense star formation activity (Puget et al. 1996; Dwek et al. 1998). Furthermore, the Infrared Space Observatory (ISO), in combination with the availability of new ground facilities such as SCUBA on the JCMT, has facilitated discovering of numerous high- $z$ galaxies with enhanced IR emission (e.g. Elbaz et al. 1999; Smail et al. 2000; Barger et al. 2000).

Silva et al. (1998) first introduced the concept of ageselective obscuration to explain the features of the observed 
spectral energy distribution (SED) of star forming galaxies, from normal spirals to dust obscured starbursts, from the UV to the sub-mm. In this model, young stars are supposed to originate within molecular clouds and correspondingly their light is attenuated more than that of older stars, that have already lost their parental cloud. The UV light in many starbursts is thus dominated by the older stars rather than by the younger populations. With the same assumptions, Granato et al. (2000) reproduced the observable properties of local galaxies (in particular the IRAS luminosity function), working within the context of structure formation through hierarchical clustering, which has successfully dealt with a wide range of observations on large scale structure and microwave background anisotropies. They showed that the concept of age-selective obscuration could explain the difference between the galactic extinction law and the attenuation law observed in starburst galaxies (Calzetti et al. 1994).

Poggianti et al. (2001) have recently investigated the optical spectra of very luminous infrared galaxies to constrain the recent history of SF and the dust extinction characteristics of various stellar populations. They have found that the most plausible explanation for their unusual combination of strong $\mathrm{H} \delta$ absorption and moderate [OII] emission is again age-selective extinction. Indeed HII regions (wherein the [OII] emission originates) are highly embedded and thus are affected by a greater extinction compared to the older stellar populations that are responsible for the Balmer absorption. Under standard assumptions for the IMF, the SFR derived from the fit of the optical spectrum (continuum, absorption and emission lines) may account for a small fraction of the FIR emission. Moreover, even complemented with the information on the FIR flux, the optical-UV spectrum is not enough to identify unique solutions. Further evidence in this direction is provided by recent observations of UV properties of ULIRGs (Goldader et al. 2002).

These studies underline the intrinsic difficulty of evaluating the properties of massive starbursts only from their UV, optical and even NIR properties and the natural way out from this impasse seems provided by studies at longer wavelengths. The capability of FIR and radio spectral regions to reveal otherwise hidden complex phenomena in star forming galaxies is supported by the existence of a "miraculous" correlation between their properties in these spectral windows. The FIR/radio correlation is locally well established over a significant range of luminosity, from normal spirals to the most extreme ULIRGs and its small scatter states the universal proportions with which energy is radiated away at IR and radio wavelengths. In spite of its obscure nature, its utility appears in several aspects, beside being a confirmed tool within the manifold of star formation indicators. For instance, the validity of the FIR/radio correlation has been recently confirmed up to redshift $\simeq 1.3$ (Garret 2001) and it is widely extrapolated much beyond, to estimate the redshift of more distant objects (e.g. Carilli \& Yun 2000). Also, deviations from the correlation observed toward the central regions of rich clusters of galaxies, where a significant fraction of star forming galaxies show a radio excess, are used to trace the effect of the hot intracluster medium on their galactic magnetic field (Gavazzi \& Jaffe 1986; Miller \& Owen 2001).
So far there have been many attempts to explain the FIR/radio correlation but all have sooner or later invoked a fine tuning of the relevant physical properties, such as the intensity of the radiative and magnetic energy density (e.g. Lisenfeld et al. 1996). In this paper we revisit this correlation by combining our spectrophotometric code GRASIL, particularly suited to the study of the IR properties of dusty galaxies, with a new model of radio emission. The latter essentially follows the recipes by Condon (1992), but after a careful assessment of the validity of one of its basic assumptions, namely the proportionality between the non-thermal (NT) radio emission and the core-collapse supernova (CCSN) rate. This fact renders the FIR/radio correlation robust and we provide, for the first time, a simple explanation of its universality. For the same reason we show that deviations are to be expected during and soon after the starburst episode, and we suggest that they can help in constraining the star formation history of these galaxies, something that cannot be done with optical, NIR and even FIR observations.

In Sect. 2 we briefly describe our population synthesis code for dusty galaxies. Section 3 is devoted to the new model of radio emission. In Sect. 4 we describe our calibration of the NT radio emission model and obtain new relations for the SFR against radio emission for the case of quiescent galaxies. In Sect. 5 we analyse infrared and radio properties of starburst galaxies. We show that the different fading times of FIR and radio emissions may be used to reach a time resolution of a few tens of Myr, which is impossible relying only on the UV-FIR. In Sect. 6 we introduce a new diagnostic tool, the FIR/radio $(q)$ vs. radio spectral slope diagram, which potentially allows the determination of the evolutionary status of a starburst in the absence of good radio spectral coverage. We examine the location of an observed sample of compact ULIRGs and discuss whether this diagram may also provide a quantitative estimate of the threshold between AGN and star formation powered ULIRGs. In Sect. 7 we analyse the evolution in the post-starburst phase and suggest that radio excess is actually an indication of the occurrence of this phase rather than an environmental effect. In Sect. 8 we discuss the impact of these new findings on the determination of the redshift of SCUBA sources, a method that relies on the FIR/radio correlation. Section 9 is devoted to our conclusions.

\section{Infrared emission}

Infrared emission is calculated with GRASIL, a code designed to perform population synthesis in presence of dust (Silva et al. 1998). In brief, the star formation history, the metal enrichment and the current gas fraction are provided by a chemical evolution code. Stars and dust are distributed either in a disk or in a bulge or both, including high density clumps, the molecular clouds. Young stars are supposed to originate within molecular clouds and to leave them in a characteristic time scale $t_{\mathrm{esc}}$. The volume emissivity is computed by considering the light of young stars, absorbed by the molecular clouds, and the light of older populations, both propagated through the diffuse dust component. The code has been thoroughly tested against observations (e.g. Silva et al. 1998; Granato et al. 2000). 
Table 1. Parameters for a disk galaxy model. See text for details.

\begin{tabular}{cccccc}
\hline \hline$v$ & $k$ & $t_{\text {inf }}$ & $x$ & $M_{\text {Low }}$ & $M_{\mathrm{UP}}$ \\
\hline $0.5 \mathrm{Gyr}^{-1}$ & 1 & $9 \mathrm{Gyr}$ & 1.35 & $0.15 M_{\odot}$ & $120 M_{\odot}$ \\
\hline
\end{tabular}

For a given star formation history, gas fraction and metallicity, one of the parameters that largely affects the FIR emission is the escape time $t_{\mathrm{esc}}$. Silva et al. (1998) and Granato et al. (2000) have shown that the UV, optical and FIR properties of local spirals are well reproduced with a typical $t_{\mathrm{esc}} \simeq 3 \mathrm{Myr}$. Normal star forming regions are also characterized by a moderate visual attenuation $A_{\mathrm{V}} \simeq 1 \mathrm{mag}$. In the case of dusty starbursts, the slope of the UV continuum (Meurer et al. 1999) indicates large obscuration times. Silva et al. (1998) and Granato et al. (2000) were able to reproduce the SEDs, from the UV to the FIR, with a $t_{\text {esc }}$ in excess of several Myr and a compact geometry with a characteristic radius of a fraction of a kpc. Similar obscuration times seem to be required to interpret the optical spectra of very luminous infrared galaxies (Poggianti et al. 2001).

We have considered as representative of a normal spiral a model with the parameters shown in Table 1. We adopt a Schmidt type star formation law, i.e. SFR $=v M_{\mathrm{g}}^{k}$, with infall of gas with primordial composition in a time scale $t_{\text {inf }}$, and a Salpeter initial mass function (IMF) (slope $x=1.35$ in mass), from $0.15 M_{\odot}$ to $120 M_{\odot}$. For starburst galaxies we superimpose an exponentially decreasing burst $\left(t_{\mathrm{b}}=10,15,25\right.$, $50 \mathrm{Myr}$ ) of star formation at an epoch of $11.95 \mathrm{Gyr}$, with the same IMF. The mass of stars formed during the burst is set to $10 \%$ of the disk mass. Our main conclusions are not affected by the details of these choices.

\section{Radio emission}

A clear picture of radio emission from normal galaxies is still missing, particularly for the often dominant non-thermal component. Indeed, it is well known that the intensity of the thermal component is tightly related to the number of $\mathrm{H}$ ionizing photons, $Q(\mathrm{H})$, and scales as $\approx v^{-0.1}$ (Rubin 1968). Computing HII region models with CLOUDY (Ferland 1996), for different mass $\left(10^{4} M_{\odot}\right.$ to $\left.10^{5} M_{\odot}\right)$, metallicity $(Z=0.008,0.02$ and 0.05 ) and age ( 1 to $10 \mathrm{Myr}$ ) of the ionizing cluster, and for electron densities of $10^{1}$ to $10^{3} \mathrm{~cm}^{-3}$ and inner radii of the nebula of 10 and $100 \mathrm{pc}$, we have obtained the following average relation at $1.49 \mathrm{GHz}$ (see Panuzzo et al. 2002 for details of the inclusion of nebular emission in GRASIL):

$\frac{L_{v}^{\mathrm{T}}}{\mathrm{erg} / \mathrm{s} / \mathrm{Hz}} \simeq \frac{Q(\mathrm{H})}{5.495 \times 10^{25}}\left(\frac{T_{\mathrm{e}}}{10^{4} \mathrm{~K}}\right)^{0.45}\left(\frac{v}{1.49 \mathrm{GHz}}\right)^{-0.1}$.

It is then straightforward to obtain $Q(\mathrm{H})$ and the intensity of thermal radio emission from simple stellar populations.

On the other hand, little is known about the source of the non-thermal emission which, in normal star forming galaxies may account $90 \%$ of the radio emission (Condon 1992). Observations indicate that FIR and radio emission are strongly correlated over a wide range of IR luminosities. At $1.49 \mathrm{GHz}$ (Sanders \& Mirabel 1996):

$q=\log \frac{F_{\mathrm{FIR}} /\left(3.75 \times 10^{12} \mathrm{~Hz}\right)}{F_{v}(1.49 \mathrm{GHz}) /\left(\mathrm{W} \mathrm{m}^{-2} \mathrm{~Hz}^{-1}\right)} \simeq 2.35 \pm 0.2$

where $F_{\text {FIR }}=1.26 \times 10^{-14}\left(2.58 S_{60 \mu \mathrm{m}}+S_{100 \mu \mathrm{m}}\right) \mathrm{W} \mathrm{m}^{-2}$, with $S_{60}$ and $S_{100}$ in Jy. This correlation suggests that NT emission is related to the recent star formation and the most likely mechanism is synchrotron emission from relativistic electrons accelerated into the shocked interstellar medium, following CCSN explosions. But the poor knowledge of the accelerating mechanism hinders any quantitative prediction of this phenomenon which can only be formulated in an empirical way (Condon 1992). The observed average luminosity per supernova event can be estimated with the ratio between NT radio emission and CCSN rate $\left(v_{\mathrm{CCSN}}\right)$ in our Galaxy (e.g. Condon $\&$ Yin 1990). With $v_{\mathrm{CCSN}} \simeq 0.015$ (Turatto, private communication) and after converting $L_{0.4 \mathrm{GHz}} \simeq 6.1 \times 10^{21} \mathrm{~W} \mathrm{~Hz}^{-1}$ (Berkhuijsen 1984) to $1.49 \mathrm{GHz}$ by assuming a radio slope of $\alpha \equiv-\frac{\mathrm{d} \log S_{v}}{\mathrm{~d} \log v}=0.8$, we obtain

$E_{1.49}^{\mathrm{NT}}=\frac{L_{1.49 \mathrm{GHz}}^{\mathrm{NT}} /\left(10^{30} \mathrm{erg} \mathrm{s}^{-1} \mathrm{~Hz}^{-1}\right)}{v_{\mathrm{CCSN}} / \mathrm{yr}^{-1}} \simeq 1.44$

It is still a matter of debate as to why the FIR/radio correlation is unaffected by the dependence of the electron lifetime and luminosity on the magnetic and radiation density fields, which may change significantly in different environments.

Lisenfeld et al. (1996) claimed that, in normal star forming galaxies, the existence of the FIR/radio correlation requires a correlation between the radiation and magnetic energy density fields. However the radiation field changes dramatically in starburst galaxies, and there must be a significant fine tuning between the parameters regulating the intensity of the two fields, because they must scale in such a way that they give rise to the FIR/radio correlation and, at the same time, they must prevent inverse Compton to dominate over synchrotron losses.

As a possible way out of this conundrum, we suggest here that a FIR/radio correlation originates because synchrotron electron lifetimes are shorter than the fading time of the CCSN rate. Assuming that cosmic ray electrons are injected during the adiabatic phase of SN explosions $\left(t \leq 10^{4}-10^{5} \mathrm{yr}\right.$ ), i.e. in a characteristic time scale which is short compared to the star formation time scale, the bolometric synchrotron luminosity at an epoch $T$ is given by

$L^{\mathrm{NT}}=\int_{0}^{\min \left(T, \tau_{\mathrm{s}}^{\mathrm{el}}\right)} v_{\mathrm{CCSN}}(T-t) l^{\mathrm{NT}}(t) \mathrm{d} t$

where $t$ is the lookback time, $l^{\mathrm{NT}}(t)$ is the NT luminosity of the injected electrons after a time $t$ has elapsed, $T$ is the age of the galaxy and $\tau_{\mathrm{s}}^{\mathrm{el}}$ is the lifetime of electrons against synchrotron losses and depends on the intensity of the magnetic field. For normal spiral galaxies $\tau_{\mathrm{s}}^{\mathrm{el}}(\ll T)$ may be as large as several $10^{7} \mathrm{yr}$ but, since the star formation rate is almost constant over the last Gyr, the above integral becomes

$L^{\mathrm{NT}}=v_{\mathrm{CCSN}} \int_{0}^{\tau_{\mathrm{s}}^{\mathrm{el}}} \frac{\mathrm{d} E}{\mathrm{~d} t} \mathrm{~d} t=v_{\mathrm{CCSN}} E^{\mathrm{el}}$ 
where we put $l^{\mathrm{NT}}(t)=\mathrm{d} E / \mathrm{d} t$. Thus the bolometric NT luminosity scales linearly with the SN rate and the proportionality constant is the injected energy of the electrons per SN $\left(E^{\mathrm{el}}\right)$.

In the case of starburst galaxies the $\mathrm{SN}$ rate cannot be considered constant over such a large time scale. However also $\tau_{\mathrm{s}}^{\mathrm{el}}$ must be much shorter: to avoid significant losses from inverse Compton on the intense stellar radiation field $\tau_{\mathrm{s}}^{\mathrm{el}} \ll 1-0.1 \mathrm{Myr}$ (Condon 1992). Thus we may still make use of the approximation in Eq. (5), because we may consider $v_{\mathrm{CCSN}}$ almost constant over such a small time scale.

In brief, the NT radio luminosity of a galaxy is proportional to the integral of the synchrotron power over the electron lifetime, and an increase of the former in a larger magnetic field is compensated by a shortening of the latter.

Since both the SN rate and the FIR emission are strictly related to the recent star formation rate, our justification of the validity of Eq. (5) in very different environments, explains why the FIR/radio correlation is so robust. No fine tuning is necessary, apart from the requirement that the magnetic field is large in starburst galaxies, which, by itself, is an independent observational fact.

The considerations above apply to the bolometric radio luminosity, but what is actually measured is the specific luminosity $L_{v}^{\mathrm{NT}}$. However, under plausible assumptions, also $L_{v}^{\mathrm{NT}}$ scales linearly with the SN rate, with the only dependence on environmental conditions being an almost vanishing one on the magnetic field. This is a consequence of the observed spectral index of the NT radio emission.

Indeed, the diffusion-loss equation (e.g. Longair 1994) for the time evolution of the number density of relativistic electrons per unit energy interval $N(E, t)$ is

$\frac{\mathrm{d} N(E, t)}{\mathrm{d} t}=\frac{\mathrm{d}}{\mathrm{d} E}[b(E) N(E, t)]+Q(E, t)+D \nabla^{2} N(E, t)$

where $b(E)$ is the rate at which particles lose energy, $Q(E, t)$ is the rate at which electrons are injected in the system per unit volume, time and energy interval (the source term) and $D$ is the diffusion coefficient. We assume that the distribution of sources of fresh electrons (i.e. the SNae) is sufficiently uniform and extended to make negligible the diffusion term, and that each SN event injects high energy electrons with a power law energy spectrum $\propto E^{-p}$, so that the source term can be written as

$Q(E, t)=k \frac{v_{\mathrm{CCSN}}(t)}{V} E^{-p}$

where $k$ and $p$ are constants (depending on the detailed physics of $\mathrm{SN}$ explosion) and $V$ is the volume of the system. Also, we are interested in stationary solutions $\mathrm{d} N / \mathrm{d} t=0$, that is we consider electron energies at which the lifetime against radiative losses is short compared to the typical time scale for variations of $v_{\mathrm{CCSN}}(t)$. The solution of Eq. (6) is then simply

$N(E)=\frac{k v_{\mathrm{CCSN}} E^{-(p-1)}}{V(p-1) b(E)}$

where $b(E)$ is the rate at which particles lose energy. If the dominant loss mechanism is synchrotron radiation,

$b(E)=-\frac{\mathrm{d} E}{\mathrm{~d} t}{ }_{\text {synch }}=\frac{4}{3} \sigma_{\mathrm{T}} c \gamma^{2} \frac{B^{2}}{8 \pi}$.
In this case

$N(E)=\frac{A k v_{\mathrm{CCSN}}}{V} \frac{E^{-(p+1)}}{B^{2}}$

where the multiplicative factor $A$ depends only on $p$ and fundamental constants: electrons with a sufficiently short lifetime have a power law energy distribution, with an index steeper by 1 than the injected distribution.

On the other hand, the synchrotron luminosity of an optically thin source with a random magnetic field and electrons having a power law energy distribution $N(E)=C E^{-q}$ is

$L_{v}^{\mathrm{NT}}=j_{v, \text { synch }} V=C D(q) V B^{\frac{q+1}{2}} v^{-\frac{q-1}{2}}$

$D(q)$ depends only on $q$ and fundamental constants. This equation with $C=A k v_{\mathrm{CCSN}} / V B^{2}$ and $\alpha=(q-1) / 2$ becomes

$L_{v}^{\mathrm{NT}}=v_{\mathrm{CCSN}}\left(A k D(p) B^{\alpha-1} v^{-\alpha}\right)$

which shows that also the specific luminosity is proportional to the $\mathrm{SN}$ rate, with a proportionality factor which, given the observed spectral index of radio emission $\alpha \approx 1$, has only a very weak dependence on the magnetic field. It may be useful to note explicitly that the above formula integrated over $v$ yields again the result expressed by Eq. (5), namely that the bolometric NT luminosity does not depend on $B$. This is because the limit of integration in frequency are those corresponding to the limits in energy within which the power law electron distribution applies, and the link between the two is $v \propto B \times E$.

The crucial assumptions in this derivation are that the $\mathrm{SN}$ rate is constant over time scales of the order of the lifetimes of relevant electrons, and that the dominant electron energy loss is synchrotron emission, as already discussed.

\subsection{The contribution of radio Supernova Remnants}

Before discussing how to calibrate Eq. (5), we consider the possible sources of non-thermal radio emission in our own Galaxy.

We have checked that, among the identified galactic sources, only radio Supernova Remnants (SNR) may provide a significant contribution to the NT radio emission. Other sources like the pulsars themselves and the bubbles of NT radio emission associated with X-ray binaries provide a negligible contribution. However SNRs, while being the obvious most appealing sources, cannot be responsible of the bulk of NT radio emission in normal galaxies for two reasons. First, their spectrum has a characteristic radio slope $L_{v} \propto v^{-\alpha}$ with a broad range of $\alpha$ between 0.2 and 0.5 (Gordon et al. 1999) which is, on average, less than that characteristic of normal galaxies ( $\alpha \simeq 0.8$ ). Second, one may easily show that they cannot supply more than about $5 \%$ of the total NT luminosity, or, equivalently, the SN rate needed to reproduce the NT radio emission of the Galaxy is more than one order of magnitude larger than observed (see e.g. Condon 1992 and references therein).

In fact, because the typical lifetime of a SNR is of a few $10^{4} \mathrm{yr}$, we may estimate the contribution of a population of SNRs originated by an instantaneous burst of star formation, by applying the fuel consumption theorem of post main sequence evolutionary phases (Renzini \& Buzzoni 1986). 
Indeed short evolutionary phases beyond the main sequence provide an integrated luminosity

$L^{\mathrm{SSP}}=\phi\left(m_{\mathrm{d}}\right) \frac{\mathrm{d} m_{\mathrm{d}}}{\mathrm{d} t} \int_{t_{\mathrm{beg}}}^{t_{\mathrm{end}}} l_{\mathrm{d}}(t) \mathrm{d} t$

where $m_{\mathrm{d}}$ is the dying mass ( $\left.\simeq M_{\text {Turn-off }}\right), l_{\mathrm{d}}(t)$ is the luminosity evolution within the phase, $\phi\left(m_{\mathrm{d}}\right)$ is the IMF, $\frac{\mathrm{d} m_{\mathrm{d}}}{\mathrm{d} t}$ is the time derivative of the dying mass, and the integral refers to the particular phase. Altogether the quantity $\phi\left(m_{\mathrm{d}}\right) \frac{\mathrm{d} m_{\mathrm{d}}}{\mathrm{d} t}$ provides the evolutionary flux, namely the rate of dying stars of the given population.

The luminosity evolution of a single SNR may be derived by combining the observed surface brightness-diameter $(\Sigma-D)$ relation at $408 \mathrm{MHz}$

$\Sigma\left(\mathrm{W} \mathrm{m}^{-2} \mathrm{~Hz}^{-1} \mathrm{sr}^{-1}\right) \simeq 10^{-15} D_{\mathrm{pc}}^{-3}$

with the time evolution of the linear diameter of a SNR (Clark \& Caswell 1976)

$D_{\mathrm{pc}} \simeq 0.43 E_{50}^{1 / 5} n^{-1 / 5} t^{2 / 5}$.

It follows that

$l\left(\mathrm{erg} \mathrm{s}^{-1} \mathrm{~Hz}^{-1}\right) \simeq 3.55 \times 10^{26} D_{\mathrm{pc}}^{-1}$

and the evolution of the luminosity of a SNR (at $1.49 \mathrm{GHz}$, assuming a slope $\alpha=0.3$ ) is (see e.g. Condon 1992)

$\frac{l_{1.49}(t)}{\mathrm{erg} / \mathrm{s} / \mathrm{Hz}} \simeq 10^{26} E_{50}^{-1 / 5} n^{1 / 5} t^{-2 / 5}$.

The integrated contribution of the population of SNRs from an instantaneous burst of star formation, at an age $t$, is then

$\frac{L_{1.49}^{\mathrm{SSP}}}{\mathrm{erg} / \mathrm{s} / \mathrm{Hz}} \simeq 1.7 \times 10^{26} E_{50}^{-1 / 5} n^{1 / 5} \tau_{\mathrm{c}}^{3 / 5} \phi\left(m_{\mathrm{d}}\right) \frac{\mathrm{d} m_{\mathrm{d}}}{\mathrm{d} t}$

where $E_{50}$ is the SN blast energy in units of $10^{50} \mathrm{erg}, n\left(\mathrm{~cm}^{-3}\right)$ is the ambient particle density and $\tau_{\mathrm{c}}$ is the lifetime of the SNR in yr. Note that $m_{\mathrm{d}}$ is a function of time, but it is almost constant during the considered phase.

The lifetime of a SNR is usually associated with its adiabatic phase (Condon 1992)

$\tau_{\mathrm{c}} \simeq 2 \times 10^{4} E_{50}^{4 / 17} n^{-9 / 17} \mathrm{yr}$

thus

$\frac{L_{1.49}^{\mathrm{SSP}}}{\mathrm{erg} / \mathrm{s} / \mathrm{Hz}} \simeq 0.6 \times 10^{29} n^{-2 / 17} E_{50}^{-1 / 17} \phi\left(m_{\mathrm{d}}\right) \frac{\mathrm{d} m_{\mathrm{d}}}{\mathrm{d} t}$.

Integrating over the past SFR, $\varphi(t)$, until we still get CCSN events $\left(t \leq t_{\mathrm{CCSN}}\right)$ and after defining the current supernova rate

$v_{\mathrm{CCSN}}\left(\mathrm{yr}^{-1}\right)=\int_{0}^{t_{\mathrm{CCSN}}} \phi\left(m_{\mathrm{d}}\right) \frac{\mathrm{d} m_{\mathrm{d}}}{\mathrm{d} t} \varphi(t) \mathrm{d} t$,

we obtain for the average SNR non thermal luminosity per supernova event

$E_{1.49}^{\mathrm{SNR}}=\frac{L_{1.49}^{\mathrm{SNR}} /\left(10^{30} \mathrm{erg} / \mathrm{s} / \mathrm{Hz}\right)}{v_{\mathrm{CCSN}} / \mathrm{yr}^{-1}} \simeq 0.06 n^{-2 / 17} E_{50}^{-1 / 17}$.
This result, independent from the assumed IMF and star formation history, is also not very sensitive to the environment. It clearly shows that the contribution of SNRs is much less than required by Eq. (3) for plausible values of $n$ and $E_{50}$ (Condon 1992). In principle the lifetime of a SNR is longer than the adiabatic time and could also depend on the ambient density. Kafatos et al. (1980) quote for a hot cavity $\mathrm{SNR} \tau_{\mathrm{SNR}}=$ $1.4 \times 10^{5} \mathrm{yr}$, at which time the linear diameter is about $100 \mathrm{pc}$ and the velocity of the shock front is $300 \mathrm{~km} \mathrm{~s}^{-1}$, still capable of maintaining a shock temperature of about $10^{6} \mathrm{~K}$. However this lifetime is still one order of magnitude less than that required by the observed NT luminosity $\left(L \propto \tau^{3 / 5}\right)$ and, more important, when the diameter is larger than about $20 \mathrm{pc}$ the observed $\Sigma-D$ relation steepens considerably $\left(\Sigma \propto D^{-10}\right)$ so that, in any case, the contribution beyond $\tau_{\mathrm{c}}$ is negligible.

\section{Calibration of the NT radio emission}

In summary, in modelling the properties of the NT radio luminosity in our Galaxy we have found that the only nonnegligible discrete sources, SNRs, can provide at most $6 \%$ of the NT radio luminosity (e.g. Condon 1992). Following Condon \& Yin (1990) we have thus calibrated Eq. (5) (after accounting for the small contribution of SNRs) against the $\mathrm{SN}$ rate and synchrotron luminosity of our Galaxy. We assume for the NT radio emission

$$
\begin{aligned}
& \frac{L^{\mathrm{NT}}(v)}{10^{30} \mathrm{erg} \mathrm{s}^{-1} \mathrm{~Hz}^{-1}}= \\
& \quad\left(E_{1.49}^{\mathrm{SNR}}\left(\frac{v}{1.49}\right)^{-0.5}+E_{1.49}^{\mathrm{el}}\left(\frac{v}{1.49}\right)^{-\alpha}\right) \times \frac{v_{\mathrm{CCSN}}}{\mathrm{yr}^{-1}} .
\end{aligned}
$$

Since the NT contribution of SNRs has been evaluated in Eq. (16) $\left(E_{1.49}^{\mathrm{SNR}} \simeq 0.06\right)$ then Eq. (3) requires that $E_{1.49}^{\mathrm{el}} \simeq$ 1.38. In order to reproduce the observed average slope of the NT radio emission in normal spiral galaxies $(\simeq 0.8)$, we have adopted $\alpha \simeq 0.9$.

An independent check of this calibration is provided by the observed ratio between the FIR and radio emission in normal spirals (Eq. (2)). We have evolved several models with GRASIL for different values of the critical parameters for the chemical evolution (different age, gas infall time scale and star formation efficiency), the escape time from molecular clouds (2 Myr and 3 Myr, Granato et al. 2000) and the scale length of the dust distribution ( $3 \mathrm{kpc}$ and $4 \mathrm{kpc}$ ). The values of $q$ turn out to be quite independent from the adopted parameters and cluster around $q=2.35$, in excellent agreement with the observations. We emphasize that, in our models, the SFR depends linearly on the gas fraction (Schmidt law), while the FIR emission depends on the SFR, gas fraction and metallicity, and the SN rate on the recent SFR. The consistency between FIR emission, radio emission and supernova rate, is thus remarkable and should be considered as a successful test of the model. We have also obtained the following calibrations between radio emission and star formation rate:

$$
\begin{aligned}
& \operatorname{SFR}\left(M_{\odot} / \mathrm{yr}\right)=2.8 \times 10^{-28} L(8.4 \mathrm{GHz}) /(\mathrm{erg} / \mathrm{s} / \mathrm{Hz}) \\
& \operatorname{SFR}\left(M_{\odot} / \mathrm{yr}\right)=7.5 \times 10^{-29} L(1.49 \mathrm{GHz}) /(\mathrm{erg} / \mathrm{s} / \mathrm{Hz}) .
\end{aligned}
$$


Table 2. Starburst parameters for the SEDs in Figs. 1 and 2.

\begin{tabular}{lcccc}
\hline \hline Case & $\begin{array}{c}\text { Age } \\
(\mathrm{Myr})\end{array}$ & $\begin{array}{c}t_{\mathrm{b}} \\
(\mathrm{Myr})\end{array}$ & $\begin{array}{c}\text { Current SFR } \\
\left(M_{\odot} / \mathrm{yr}\right)\end{array}$ & $\begin{array}{c}\text { Burst Mass } \\
\left(M / M_{\text {tot }}\right)\end{array}$ \\
\hline M 82 & & & & \\
a dotted & 45 & 50 & 3.41 & 0.011 \\
b solid & 24 & 8 & 2.37 & 0.011 \\
\hline ARP 220 & & & & \\
a solid & 50 & 50 & 271 & 0.121 \\
b dotted & 40 & 30 & 280 & 0.121 \\
c dashed & 25 & 8 & 149 & 0.121 \\
\hline
\end{tabular}

These relations hold only for the case of a constant SFR for more than $100 \mathrm{Myr}$ and are in good agreement with those obtained by Carilli (2000). It is worth noticing that with a SN rate of 0.023 - the value adopted by Condon 1992 - we would have obtained a SFR calibration higher by about $50 \%$.

Before concluding this section it is worth commenting on our choice of the lower limit of the initial mass giving rise to type II SNe. It is commonly assumed that it corresponds to an initial mass of about $8 M_{\odot}$ for standard convection while it lowers to about $6 M_{\odot}$ for models with convective overshoot. However, recent investigations on the evolution between 8 and $10 M_{\odot}$ (without overshoot) indicate that the final fate of stars born in this mass range is that of a white dwarf, instead of an electron capture SN (Ritossa et al. 1996). The same fate is expected in the mass range 6 to $8 M_{\odot}$ if the overshoot scheme is adopted (Portinari et al. 1998). We have thus assumed that CCSN are produced in stars with mass $M \geq 8 M_{\odot}$ and for ages younger than $t_{\mathrm{CCSN}} \simeq 50 \mathrm{Myr}$.

\section{FIR/radio properties of starburst models}

In this section we examine the FIR and radio properties of our new models and compare them with two well-studied local starbursts, M 82 and ARP 220. To obtain a realistic starburst model, we added an exponentially decreasing burst of star formation to the underlying secular disk evolution of the spiral models used in the previous section. Table 2 summarizes the burst parameters in a few adopted models. Notice that in all models of the same galaxy, the total mass of gas converted into stars is the same (column $M / M_{\text {tot }}$ ). The corresponding GRASIL parameters have been selected according to Silva et al. (1998) and, in particular, the adopted obscuration time must be significantly larger than that characteristic of normal galaxies.

Figure 1 shows two fits to the SED of M 82, differing only in the age (45 and $24 \mathrm{Myr}$ ) and e-folding time (50 and $8 \mathrm{Myr}$ ) of the burst. The UV-optical-FIR SED of the starburst is degenerate, in the sense that it may be fitted by different set of burst parameters. Both models provide a good fit to the UV to sub-mm data. At radio frequencies however, only the short burst is able to reproduce the observations while the longer one falls significant below the observed flux. A similar example is shown in Fig. 2 for the ULIRG ARP 220. ARP 220 is not consistent with a short burst, though this could not be excluded by the inspection of UV-optical-FIR data alone. Notice that

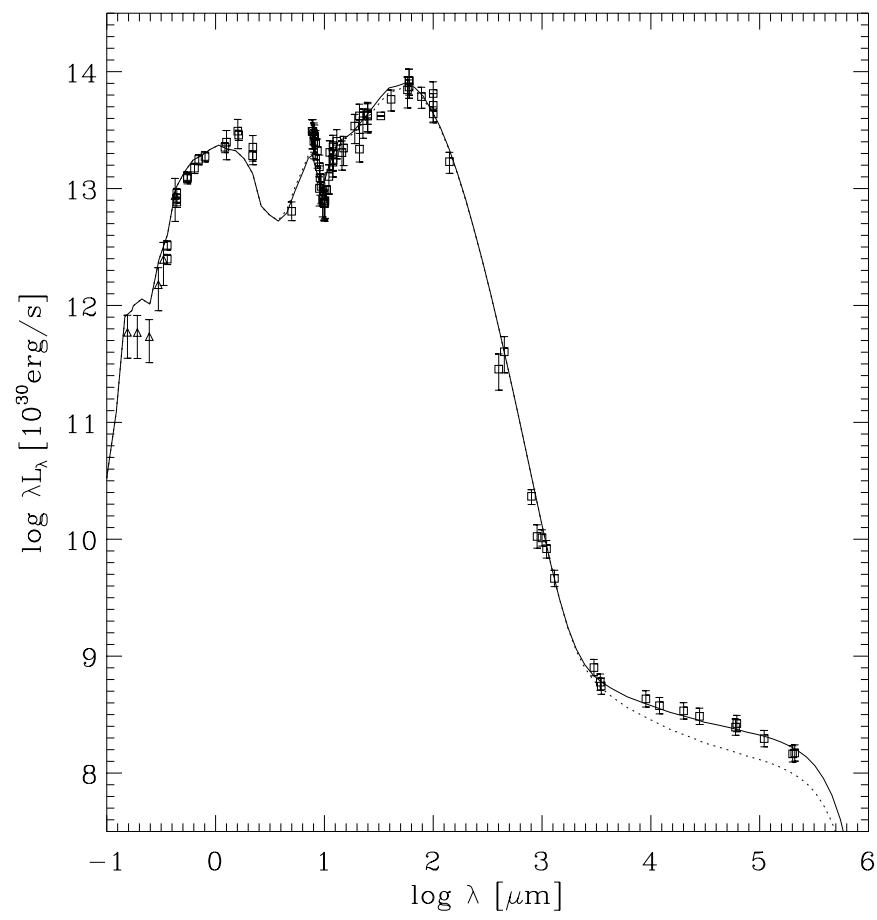

Fig. 1. M 82: two models with different age and e-folding time scale (Table 2), fit the observed SED from UV to the sub-mm. Inclusion of radio wavelengths is necessary to disentangle the two cases. For details on the models see Silva et al. (1998).

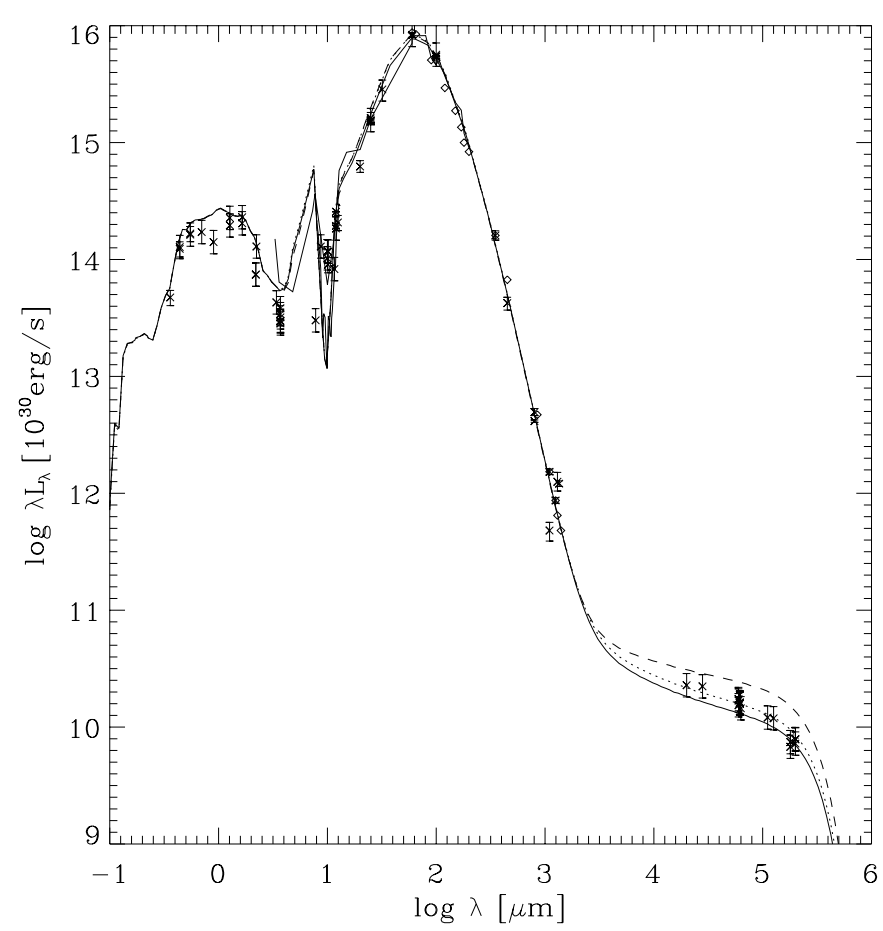

Fig. 2. Same as in Fig. 1, for three models for ARP 220.

the current SFR of the models differ by about $40 \%$ and $90 \%$ in M 82 and ARP 220, respectively.

The cases of M 82 and ARP 220 show that the inclusion of the radio emission constitutes a powerful diagnostic tool to investigate starburst galaxies. Indeed, while the UV, the FIR and the radio thermal continua are sensitive to the number of 


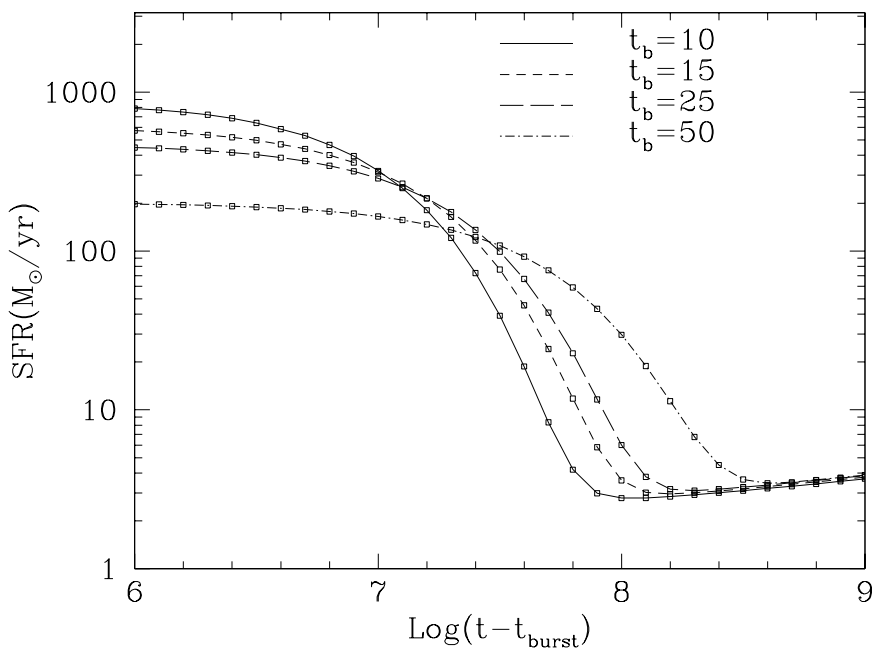

Fig. 3. The run of the star formation rate in our starburst models for different e-folding time scales, $t_{\mathrm{b}}=10,15,25$ and $50 \mathrm{Myr}$.

massive stars, the NT emission is a measure of the current CCSN rate. Thus the FIR/radio ratio is a measure of the ratio between the almost instantaneous SFR and the SFR averaged over the last few tens of Myrs. Combining the FIR and radio spectral regions is particularly important for the case of obscured starbursts, where the burst properties cannot be derived by UV, optical, NIR and even mid and far IR (continuum) observations. We ought to stress in the following, that a careful combination of FIR and Radio data may be sufficient to determine the recent star formation history even for those galaxies that are not as thoroughly observed as M 82 or ARP 220. Inspection of Figs. 1 and 2 shows that the expected value of the $q$ ratio at $1.49 \mathrm{GHz}$ and $8.44 \mathrm{GHz}$ changes by more than a factor of 2 for the different models. This suggests that deviations from the average FIR/radio correlations should be expected among obscured starbursts and actually could be used as a powerful diagnostic for the analysis of the star formation in the burst. Furthermore, also the radio slope is affected because at younger ages the relative contribution of thermal emission is larger than at older ages.

To highlight this point we have carefully analysed the evolution of selected starburst models that possibly encompass different realistic scenarios. For the star formation rate during the burst we have assumed four different e-folding time scales, $t_{\mathrm{b}}=$ 10, 15, 25 and $50 \mathrm{Myr}$. The mass of stars formed during the burst was set to $10 \%$ of the underlying disk mass. The obscuration time $t_{\mathrm{esc}}$ was set to linearly decrease with time, from $t_{\mathrm{b}}$ down to a minimum of $3 \mathrm{Myr}$, characteristic of normal galaxies. The run of the SFR of the models during the burst is depicted in Fig. 3.

Figure 4 shows the ratio between the current SFR $\left(M_{\odot} / \mathrm{yr}\right)$ and the FIR luminosity, $L_{\mathrm{FIR}}$. The latter, representing a fair measure of the luminosity between 40 and $120 \mu \mathrm{m}$, is defined in analogy with Helou et al. (1988) as $L_{\mathrm{FIR}}(\mathrm{erg} / \mathrm{s})=$ $1.257 \times 10^{-11}\left(2.58 L_{60}+L_{100}\right)$ where $L_{60}$ and $L_{100}$ are the luminosities in $\mathrm{erg} / \mathrm{s} / \mathrm{Hz}$ at 60 and $100 \mu \mathrm{m}$. In this figure, the model with $t_{\mathrm{b}}=25 \mathrm{Myr}$ has been recomputed with a fixed $t_{\mathrm{esc}}=3 \mathrm{Myr}$, corresponding to that of normal galaxies. Figure 5

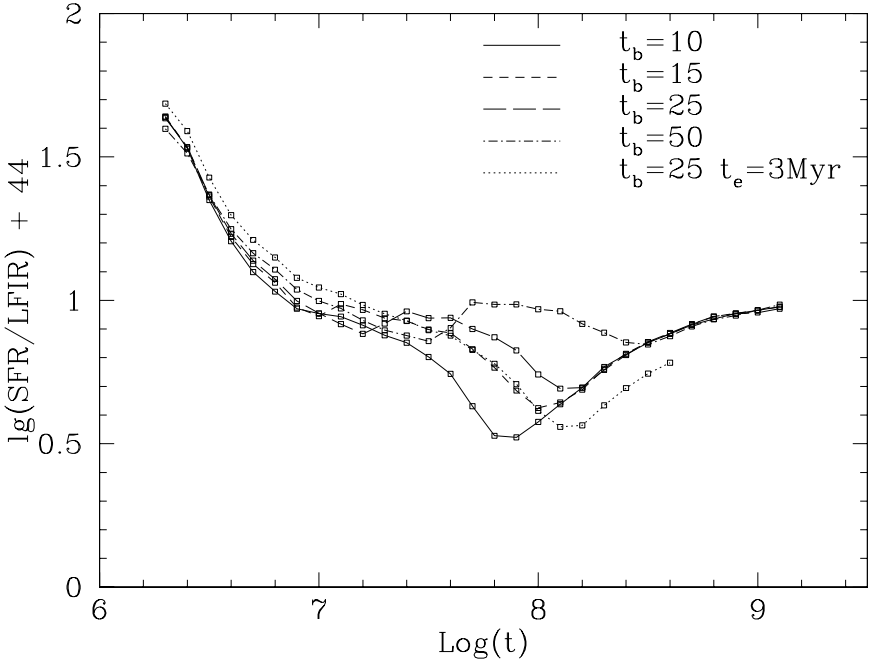

Fig. 4. Evolution of the ratio between the instantaneous star formation rate $\left(\mathrm{SFR}, M_{\odot} / \mathrm{yr}\right)$ and FIR luminosity $(\mathrm{erg} / \mathrm{s})$, see text for the definition.

shows the ratio between the SFR $\left(M_{\odot} / \mathrm{yr}\right)$ and the radio luminosity (erg/s/Hz) at $1.49 \mathrm{GHz}$ (upper panel) and at $8.44 \mathrm{GHz}$ (lower panel). Obviously, in the latter figure, $t_{\mathrm{esc}}$ is not relevant. Three main evolutionary phases can be recognised, the starburst, post-starburst and quiescent phase, characterized by different relations between either the FIR or the radio emission and the current SFR. At early times, during the starburst phase, the SFR/Flux ratio is significantly higher than the asymptotic value defined by normal star forming galaxies. At later times, when the ratio falls below the value of normal galaxies, the models enter the "post-starburst" phase. Finally, as the burst extinguishes, the ratio turns to the asymptotic value defined for normal star forming galaxies. Depending on the evolutionary status and the e-folding time of the SFR, the relations between infrared or radio emission and SFR may change by even one order of magnitude. As already anticipated, NT radio emission is more sensitive to the past star formation history than the FIR emission and the corresponding ratio shows a larger variation.

\section{FIR/radio properties of compact ULIRGs}

We have already anticipated that our models predict the existence of a correlation between the radio slope and the FIR/radio ratio, because both quantities are affected by the relative contributions of FIR, thermal and non-thermal radio emission that change during the evolution of the burst. Understanding whether the use of such correlation can provide further physical insight on the star formation process becomes, obviously, particularly relevant in the case of highly obscured starbursts such as ULIRGs, emitting the bulk of their radiation in the mid and far infrared. ULIRGs are characterized by extreme FIR luminosities exceeding the UV-optical power by even more than one order of magnitude, and are believed to be transient phases of galaxy activity associated with the dynamical interaction and merging of gas rich systems. Whether their powerful emission is of starburst origin or is due 

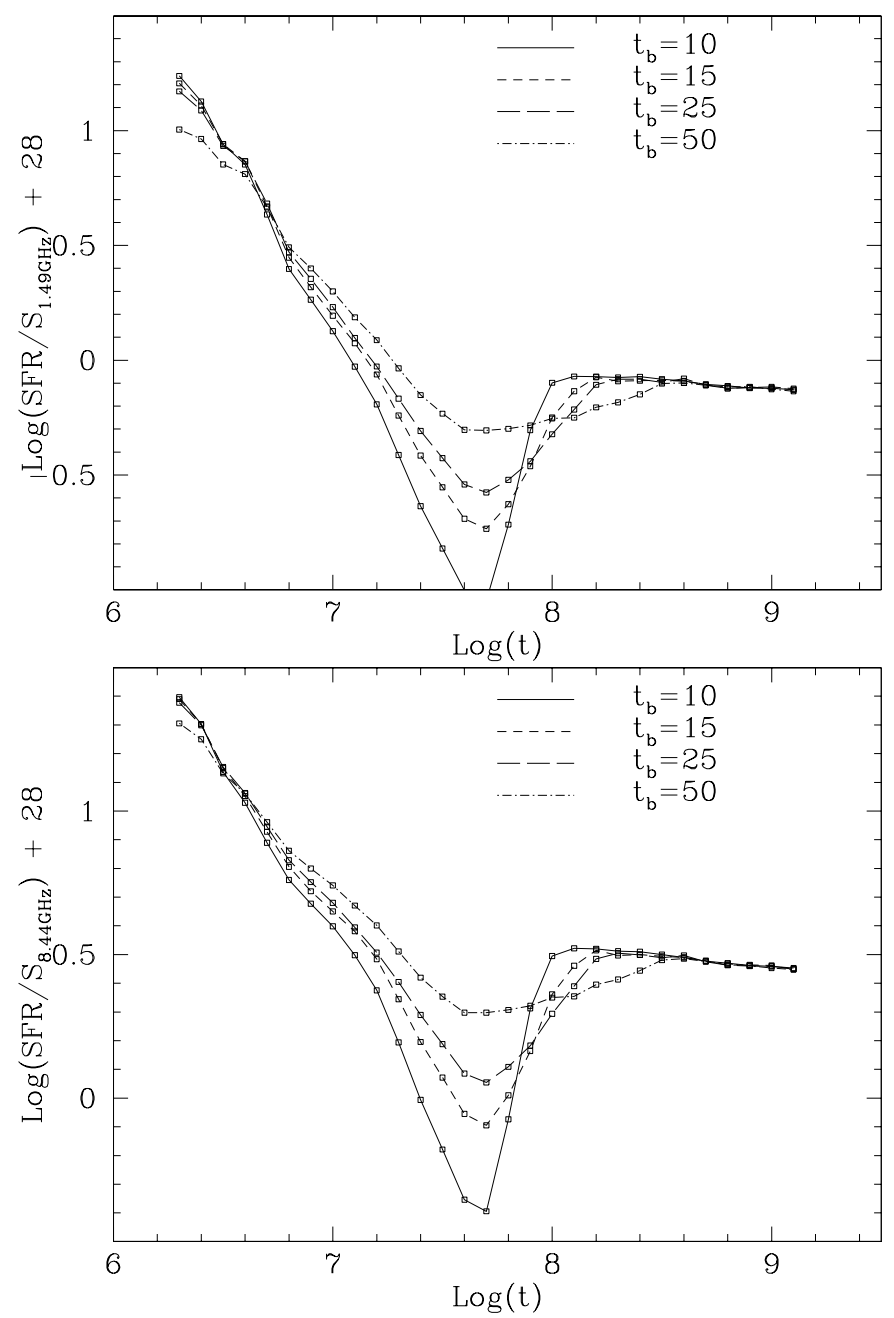

Fig. 5. Evolution of the ratio between the instantaneous star formation rate $\left(\mathrm{SFR}, M_{\odot} / \mathrm{yr}\right)$ and radio luminosity $(\mathrm{erg} / \mathrm{s} / \mathrm{Hz})$ at $1.49 \mathrm{GHz}$ (upper panel) and at $8.44 \mathrm{GHz}$ (lower panel).

to the AGN is still under debate (Sanders et al. 1988; Condon et al. 1991; Veilleux et al. 1994) but, recently, it has become clear that these objects may harbour huge compact star forming complexes and the AGN, at the same time (Rowan-Robinson 1995). However, it remains still unclear what fraction of the bolometric luminosity is eventually provided by the central monster and at what level this affects FIR and radio emission. For example, very recently Berta et al. (2002) have shown that in IRAS 19254-7245 ("The Superantennae"), the AGN is contributing about $40-50 \%$ of the MIR and FIR emission (essentially the bolometric luminosity), while in the radio it exceeds by one order of magnitude the emission from the starburst. At the same time, for IRAS 20100-4156, one of the brightest nearby objects, both the multi-wavelegth analysis (Fritz et al. 2002), spectro-polarimetry (Pernechele et al. 2002) and near-IR spectroscopy (Valdes et al. 2002) show no evidence of the AGN.

In this respect, the sample of compact ULIRGs selected by Condon et al. (1991) turns out to be particularly interesting because high resolution VLA maps suggest that the bulk of their radio continuum could be of starburst origin and several objects have been observed at both $1.49 \mathrm{GHz}$ and $8.44 \mathrm{GHz}$, so that a direct comparison in term of "observables" can be made with our starburst models. We thus isolated all the compact ULIRGs of the Condon et al. sample observed at both 1.49 GHz and 8.44 GHz, in Table 3. Optical spectroscopic classification by Veilleux et al. (1995) indeed showed that a number of the selected objects have emission line ratio characteristic of photoionization by massive stars (HII galaxies), but other show higher excitation and were classified as Liners or even Seyfert 2 galaxies. Mrk 231 is a Seyfert 1 object. A subset of the original Condon et al. sample has been subsequently mapped with VLBI by Smith et al. (1998, SLL), to establish the nature of the milli-arc second structures and in particular to obtain the brightness temperature of the compact inner core. SLL did not find significant correlations between the VLBI emission (usually $10 \%$ of the $1.6 \mathrm{GHz}$ total flux density) and other physical parameters like total radio power, FIR emission and radio slope. Perhaps more important, they have found that that optical excitation is not strictly correlated with high $T_{\mathrm{b}}$. As can be seen from their data, summarized together with the Veilleux et al. classification, in the last column of Table 3, there are HII objects showing high $T_{\mathrm{b}}$ cores and LINERs and Sey 2 (all) galaxies with central $T_{\mathrm{b}}$ consistent with a starburst origin. Furthermore, SLL were able to show that some of the high $T_{\mathrm{b}}$ cores could be explained by bright radio supernovae complexes, consistent with the star formation rates required by the FIR. These objects are marked with a lower case "a" in the last column of Table 3, while those that could not be interpreted in terms of bright SNRs by SLL, are marked by a lower case "l". Some of the high $T_{\mathrm{b}}$ objects had not enough detailed structure to allow this analysis.

According to the information provided by Veilleux et al. (1995) and SLL, we grouped all objects into four broad categories, named with the symbol reported in Col. 8 of Table 3 . The letter "H" indicates all galaxies of HII type, except for three objects for which SLL could not establish that their high $T_{\mathrm{b}}$ is compatible with RSN complexes. Analogously, Liners are indicated by the symbol " $\mathrm{L}$ " when their $T_{\mathrm{b}}$ is missing or is low, or their high $T_{\mathrm{b}}$ was found compatible with RSN complexes. Object classified as HII, Liners or AGN that do not fulfil the above requirements are indicated by the symbol "A" while, for Seyfert 2 galaxies we use the symbol "S". Finally "P" and "M" refer to ARP 220, a typical starburst galaxy and Mrk 231, a Seyfert 1 galaxy, respectively. According to this grouping, the effect of the AGN, if any, should not be significant in types $\mathrm{H}$ to $\mathrm{L}$. For example a recent comprehensive analysis of optical-NIR-FIR SED and of spectro-polarimetric data of IRAS 22491-1808, indicates that if the AGN is present in this object, it must contribute less than $5 \%$ of the total FIR emission (Fritz et al. 2002).

In Fig. 6 we show the run of the $q$ values expected from our starburst models, Eq. (2), at 1.49 and $8.44 \mathrm{GHz}$, against the FIR luminosity (now in solar units). Superimposed are the selected compact ULIRGs from the local sample of Condon et al. (1991), with the corresponding symbols listed in Table 3.

The models can be arbitrarily shifted in the horizontal direction by changing the mass of stars formed in the burst which, for the adopted normalization, corresponds to about $10^{10} M_{\odot}$. To fit the IR luminosity of the brightest compact sources we 
Table 3. Selected galaxies from the sample of compact ULIRGs by Condon et al. (1991).

\begin{tabular}{|c|c|c|c|c|c|c|c|c|}
\hline Name & $S_{1.49 \mathrm{GHz}}$ & $q$ & $S_{8.44 \mathrm{GHz}}$ & $\alpha_{1.49-8.44}$ & FIR & $N_{\mathrm{e}}^{\#}$ & Symbol $^{\natural}$ & Classification $^{\star}$ \\
\hline IRAS 10566+2448 & 46.1 & 2.55 & 14.1 & 0.68 & 11.90 & - & $\mathrm{H}$ & HII \\
\hline A11010+4107 & 28.0 & 2.54 & 10.7 & 0.55 & 11.52 & - & $\mathrm{H}$ & HII \\
\hline IRAS $12112+0305$ & 22.6 & 2.66 & 10.0 & 0.47 & 12.18 & - & $\mathrm{H}$ & HII \\
\hline UGC 08335 & 51.2 & 2.46 & 17.0 & 0.64 & 11.62 & 590 & $\mathrm{H}$ & HII \\
\hline IRAS 22491-1808 & 6.1 & 3.00 & 3.0 & 0.41 & 12.02 & - & $\mathrm{H}$ & $\mathrm{HII} \mathrm{Tb}=5.2$ \\
\hline IRAS $17132+5313$ & 28.4 & 2.46 & $8.9^{b}$ & 0.67 & 11.79 & 450 & $\mathrm{H}$ & $\mathrm{HII} \mathrm{Tb}<5$ \\
\hline UGC 04881 & 29.0 & 2.50 & 8.8 & 0.69 & 11.61 & 210 & $\mathrm{H}$ & HII Tb $>7 \mathrm{a}$ \\
\hline IRAS $01173+1405$ & 43.1 & 2.46 & 12.7 & 0.70 & 11.54 & 160 & $\mathrm{H}$ & HII Tb $>7 \mathrm{a}$ \\
\hline Mrk 331 & 67.5 & 2.51 & $21.5^{b}$ & 0.66 & 11.27 & 510 & $\mathrm{H}$ & HII $\mathrm{Tb}>7 \mathrm{a}$ \\
\hline IRAS $10173+0828$ & 8.8 & 2.92 & 5.0 & 0.28 & 11.70 & - & $\mathrm{H}$ & - \\
\hline ARP 220 & 301.1 & 2.63 & 148.0 & 0.41 & 12.11 & - & $\mathrm{P}$ & $\mathrm{HII} \mathrm{Tb}>7 \mathrm{a}$ \\
\hline IRAS 04191-1855 & 27.3 & 2.49 & 8.9 & 0.65 & 11.34 & 100 & $\mathrm{~L}$ & Liner $\mathrm{Tb}<5$ \\
\hline IRAS $08572+3915$ & 6.5 & 3.11 & 4.1 & 0.27 & 11.96 & - & $\mathrm{L}$ & Liner $\mathrm{Tb}<5$ \\
\hline IRAS 14348-1447 & 33.2 & 2.38 & 9.7 & 0.71 & 12.17 & - & $\mathrm{L}$ & Liner $\mathrm{Tb}<5$ \\
\hline IRAS 01364-1042 & 17.0 & 2.67 & 8.2 & 0.42 & 11.67 & - & $\mathrm{L}$ & Liner $\mathrm{Tb}<5$ \\
\hline UGC 08387 & 106.0 & 2.28 & 34.9 & 0.64 & 11.51 & $1220^{b}$ & $\mathrm{~L}$ & Liner \\
\hline Mrk 273 & 130.0 & 2.31 & 43.5 & 0.63 & 12.04 & - & $\mathrm{L}$ & Liner $\mathrm{Tb}>7 \mathrm{a}$ \\
\hline IRAS $03359+1523$ & 18.9 & 2.58 & 11.0 & 0.31 & 11.37 & 370 & A & HII $\mathrm{Tb}>7$ \\
\hline Mrk 848 & 46.8 & 2.38 & $12.1^{b}$ & 0.78 & 11.72 & 240 & A & $\mathrm{HII} \mathrm{Tb}>7$ \\
\hline UGC 02369 & 42.7 & 2.39 & 13.3 & 0.67 & 11.42 & - & A & HII Tb > 71 \\
\hline IIIZw035 & 39.3 & 2.58 & 19.7 & 0.40 & 11.46 & 590 & A & Liner $\mathrm{Tb}=5.4$ \\
\hline IRAS $15250+3608$ & 12.8 & 2.81 & 10.5 & 0.11 & 11.88 & - & A & Liner $\mathrm{Tb}=6$ \\
\hline UGC0bright & 146.0 & 2.10 & 52.6 & 0.59 & 11.93 & - & A & Liner $\mathrm{Tb}>71$ \\
\hline NGC 2623 & 97.8 & 2.51 & 35.5 & 0.58 & 11.47 & - & A & $\mathrm{AGN} \mathrm{Tb}>71$ \\
\hline NGC 0034 & 58.7 & 2.52 & 15.2 & 0.78 & 11.28 & - & $S$ & Sey2 $\mathrm{Tb}=5$ \\
\hline Zw475.056 & 26.0 & 2.65 & 8.2 & 0.67 & 11.37 & 190 & S & Sey $2 \mathrm{~Tb}<5$ \\
\hline IRAS 05189-2524 & 28.1 & 2.76 & 11.4 & 0.52 & 11.91 & - & S & Sey $2 \mathrm{~Tb}<5$ \\
\hline Mrk 231 & 240.0 & 2.24 & 265.0 & -0.06 & 12.35 & - & M & Sey1 $\mathrm{Tb}>7$ \\
\hline
\end{tabular}

${ }^{b}$ Uncertain value.

\# Electron density, $\mathrm{cm}^{-3}$, from Veilleux et al. (1995).

${ }^{\natural}$ Symbols adopted in Figs. 6 and 7.

${ }^{\star}$ HII, Liner, AGN, Seyfert based on Veilleux et al. (1995); $T_{\mathrm{b}}$ logarithm of the brightness temperature from VLBI data by Smith et al. (1998).

need a SFR higher by about a factor of 2 to 3, for the shorter burst. We note that the observed dispersion of the $q$ ratio is well explained by the starburst models, in particular in the lower panel, where, as we will see, the effects of free-free absorption should be negligible. The mass of the burst is however not known a priori and while Fig. 6 shows that the observed data are consistent with the sources being compact starbursts, it does not allow to obtain an estimate of the burst parameters. On the other hand, the case of M 82 and ARP 220 (Figs. 1 and 2) indicates that this is within the possibility of the model when enough observations are available.

We thus combine in Fig. 7 the FIR/radio ratio with the slope of the radio emission $\alpha$ between 1.49 and $8.4 \mathrm{Ghz}$. Both quantities are independent from the intensity of the burst. Rather, they depend on the form of the recent star formation history, so that the path in this diagram traces the evolutionary status of the starburst. The radio slope of the models changes because of the variation of the dominant source of radio emission as the starburst ages. During the first 3 to 4 Myrs only thermal emission from HII regions contributes to the radio emission, and the radio flux has a characteristic slope $\alpha \simeq 0.1$. Then CCSN explosions feed relativistic electrons into the galactic magnetic field, and NT emission steepens the spectrum toward a slope which is more typical of normal galaxies. At the same time the total radio power increases and the evolution of the $q$ ratio, though affected by the corresponding increase of the FIR emission, continuously decrease to a minimum value. At this stage the SFR has decreased significantly and the model can be considered in a post starburst phase. At even older ages the ratio increases again, but this corresponds to the very late phase where the model can no longer be considered representative of an ultra luminous galaxy. The model with $t_{\mathrm{b}}=25 \mathrm{Myr}$ and obscuration time of normal galaxies, $t_{\mathrm{esc}}=3 \mathrm{Myr}$, is not able to reproduce the observed high values of $q$, in both panels, 


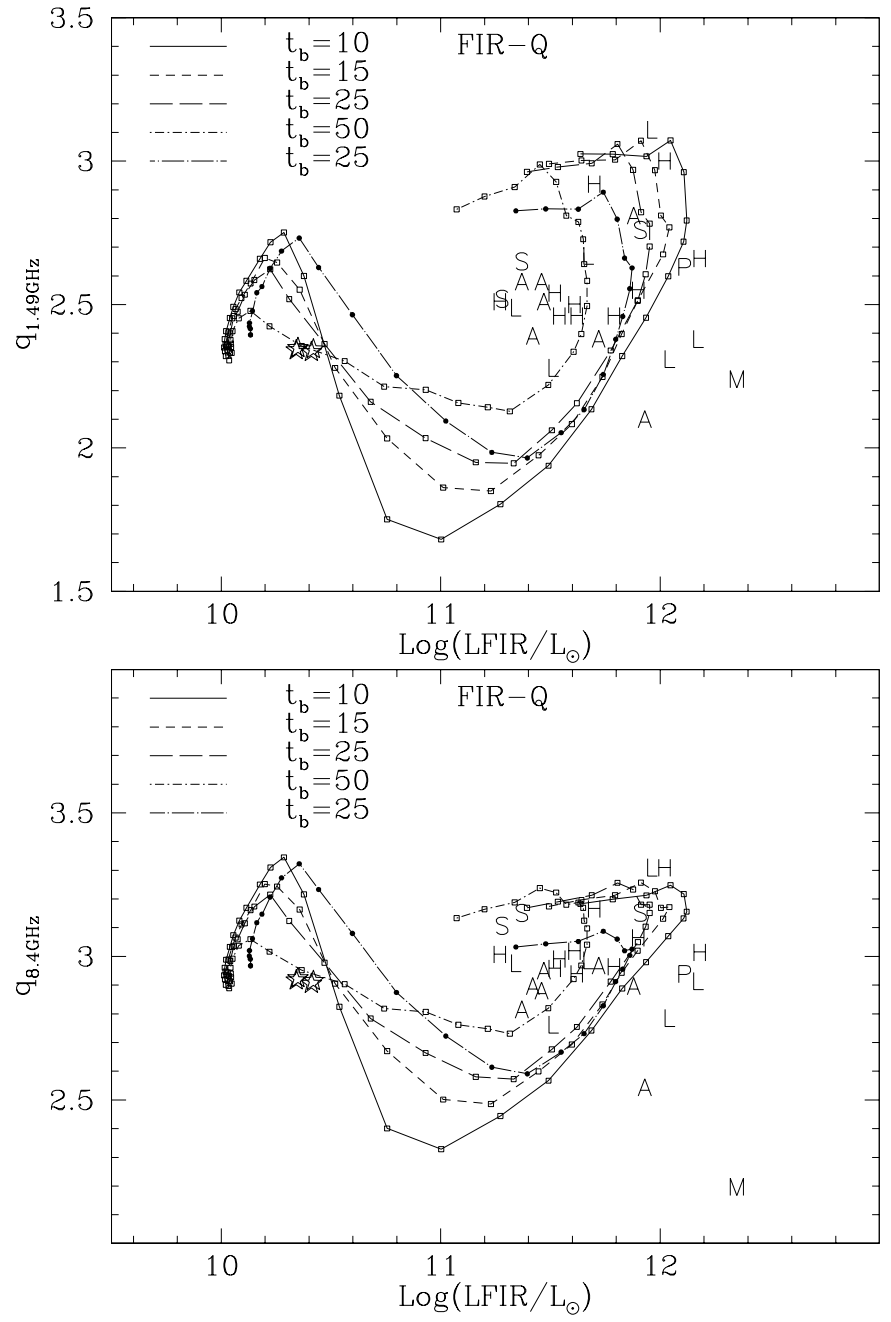

Fig. 6. Evolution of starburst models in the $q$ vs. $L_{\mathrm{FIR}}$ diagram. The upper panel is for $q$ at $1.49 \mathrm{GHz}$, the lower one is for $q$ at $8.44 \mathrm{GHz}$. Stars indicate the positions of typical models of quiescent star forming disks. The model with $t_{\mathrm{b}}=25 \mathrm{Myr}$ and $t_{\mathrm{esc}}=3 \mathrm{Myr}$ is also shown (small solid dots). Symbols refer to the subset of compact ULIRGs (Condon et al. 1991), drawn in Table 3.

confirming that the low UV flux observed in other obscured starburst (see e.g. Figs. 1 and 2) indicates that this escape time is too short. The observed data show a clear trend of increasing $q$ at decreasing slope, in the $1.49 \mathrm{GHz}$ plot, with the models being only able to delineate an upper envelope. At a higher frequency (lower panel), the trend in $q$ disappears while the models still delineate the upper envelope. A possible explanation is that part of the effect is caused by free-free absorption (see also Condon et al. 1991). At radio frequencies the optical depth of free-free absorption by a cloud with electron density $N_{\mathrm{e}}$ and size $l$, is

$\tau_{v}^{\mathrm{ff}} \simeq 8.2 \times 10^{-2} T_{\mathrm{e}}^{-1.35}\left(\frac{v}{\mathrm{GHz}}\right)^{-2.1}\left(\frac{N_{\mathrm{e}}^{2} l}{\mathrm{pc} / \mathrm{cm}^{6}}\right)$.

In order to highlight the effects of free-free absorption we have recomputed a model with optical depth $\tau_{1.49 \mathrm{Ghz}}^{\mathrm{ff}}=1$ (dot-dashed line on the left side in Fig. 7). Assuming an electron temperature $T_{\mathrm{e}}=10^{4} \mathrm{~K}$, this optical depth corresponds to an emission measure of about $6 \times 10^{6} \mathrm{pc} / \mathrm{cm}^{6}$. The latter can be achieved

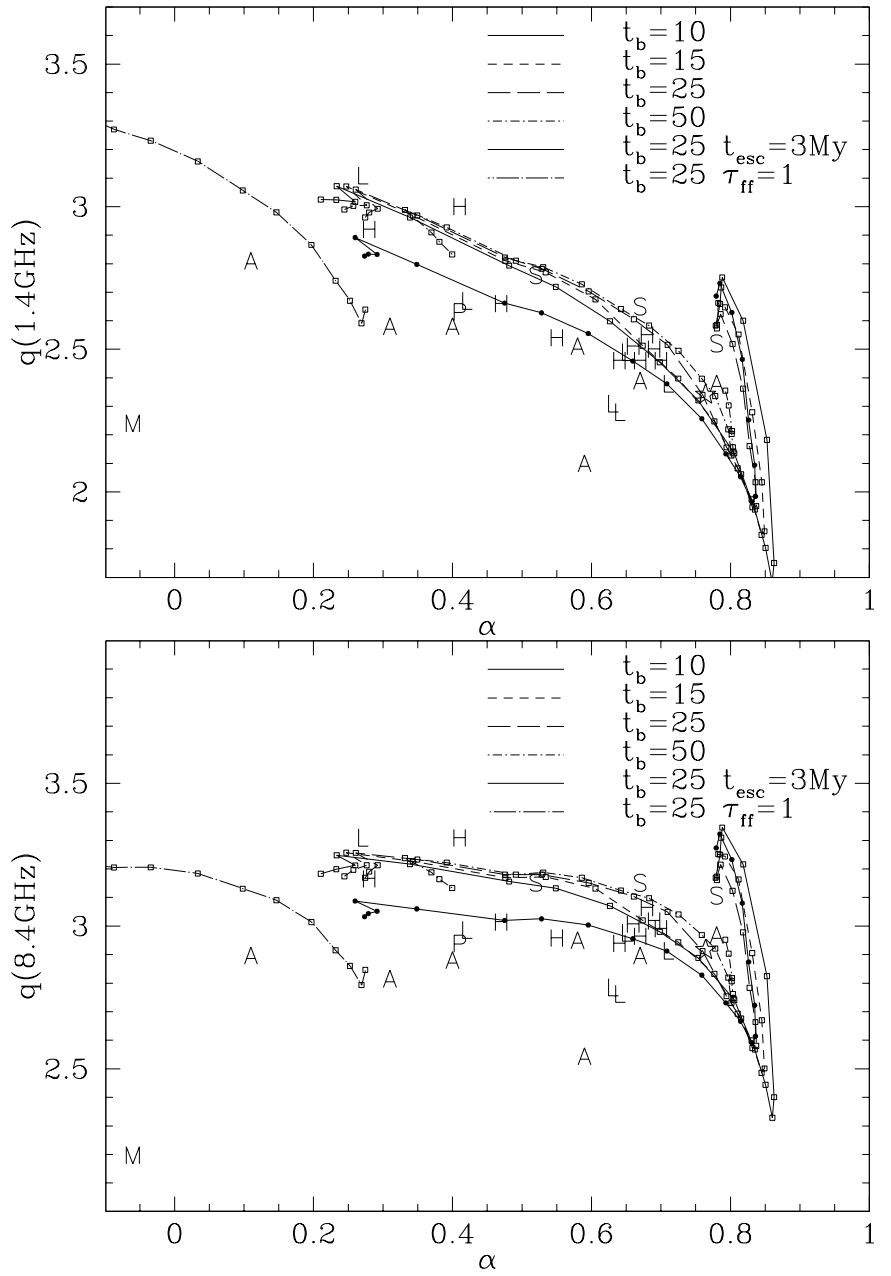

Fig. 7. Upper panel: evolution of the ratio $q$ vs. radio slope $\alpha$ between 8.4 and $1.4 \mathrm{GHz}$ for our starburst models. Characteristic starburst ages range from $\log t(\mathrm{yr})=6.3$ to 8.6 . The model with $t_{\mathrm{b}}=$ $25 \mathrm{Myr}$ has been recomputed both with a $t_{\mathrm{esc}}=3 \mathrm{Myr}$ (crossing the observed data) and by adopting a free-free absorption with $\tau_{v=1.4 \mathrm{GHz}}=1$ (model on the left side up to its minimum value of $q$ ). Lower panel: same for $v=8.4 \mathrm{GHz}$. Note that $q$ is no more affected by free-free absorption. Symbols refer to the subset of compact ULIRGs (Condon et al. 1991), drawn in Table 3.

by considering $2.5 \times 10^{8} M_{\odot}$ within 250 pc or $7.8 \times 10^{8} M_{\odot}$ within $390 \mathrm{pc}$ of ionised gas, for an average electron density of about $N_{\mathrm{e}}=150 \mathrm{~cm}^{-3}$ or $N_{\mathrm{e}}=125 \mathrm{~cm}^{-3}$ and solar composition, respectively. The above figures compare fairly well with electron densities derived from emission line ratios by Veilleux et al. (1995), listed in Table 3. They are consistent both with the fact that the selected ULIRGs are compact radio sources with typical sizes less than $1 \mathrm{kpc}$ and possibly as low as a few hundred pc (Soifer et al. 2000), and that the total mass involved in our starburst models is of the order of $10^{10} M_{\odot}$.

The distribution of the data in Fig. 7 also renders quite unlikely the possibility that the slope variation is due to an increasing importance of the electron cooling by inverse Compton. Indeed it could be that in ULIRGs with the lower $\alpha$ electrons cool down by inverse Compton on the stellar radiation field: in those objects only thermal emission would be present at radio wavelengths. Not only would this again require 
a fine tuning of the two cooling processes, in order to give rise to a distribution of slopes between 0.1 and 1 but, and more important, it would be difficult to explain the absence of the trend at the higher frequency. This re-inforces our interpretation in terms of free-free absorption because, given the frequency dependence (Eq. (18)), this effect should not affect the value of $q$ at $8.44 \mathrm{GHz}$. The quantity $q_{8.44 \mathrm{GHz}}$, which still shows a range of about $0.6 \mathrm{dexp}$, can be considered as a genuine measure of the age of the compact starbursts. Unfortunately, in this diagram, the slope is still affected by free-free absorption, and it is not possible from the above data alone to identify precisely the evolutionary status of the ULIRGs. However, our models suggest that, observing ultra luminous galaxies at frequencies between $8.44 \mathrm{GHz}$ and $23 \mathrm{GHz}$ would possibly constitute a powerful tool to investigate on the recent star formation history of obscured starbursts.

From the upper panel of Fig. 7 it is also evident that there is no sharp threshold value of $q_{1.49 \mathrm{GHz}}$ that can be safely used to delineate a separation between star formation and AGN powered ULIRGs. There is a tendency for objects classified " $\mathrm{A}$ " to occupy the lower boundary allowed by starburst models but, even in the case of the Seyfert 1 galaxy Mrk 231, the brightest object in our sample, it would be difficult to exclude a starburst origin based only on its $q_{1.49 \mathrm{GHz}}$. However its location in the corresponding $q$-slope diagram is not matched by any of our starburst models and, by looking at the higher frequency data $q_{8.44 \mathrm{GHz}}$, it appears to be at least three times more radio powerful (relative to the FIR) than all the other sources and than allowed by our models in the starburst phase. Is this an indication that the parameters plotted in Fig. 7 (possibly making use of a higher frequency to avoid free-free contamination of the radio slope) could be used as a diagnostic diagram to disentangle a starburst from an AGN? And, in this case, why are all the Seyfert 2 galaxies in our sample populating only the upper envelope of starburst models? From the above plot we may only conclude that a minimum value of $q_{1.49 \mathrm{GHz}}$ for a starburst powered source is around 1.8, and this may set a threshold value below which another source of radio emission has to be invoked. One should consider however that at this frequency the nuclear engine may be masked by free-free absorption. At $8.44 \mathrm{GHz}$ free-free absorption should be less important and a fair threshold for star formation dominated ULIRGs is $q_{8.44 \mathrm{GHz}}=2.5$.

\section{The post starburst phase}

In a recent comprehensive analysis of the FIR/radio correlation in nearby Abell clusters, Miller \& Owen (2001) found a statistically significant excess of star forming galaxies with enhanced radio emission relative to the FIR, toward the cluster centres. High resolution radio images have also excluded a significant AGN contribution to the radio emission in these galaxies.

There is a long standing debate on the nature of this excess of galaxies with a low value of $q_{1.49 \mathrm{GHz}}$, which is always interpreted as a radio enhancement and may reach a factor of three. Gavazzi \& Jaffe (1986) advanced the hypothesis that the radio excess is caused by the ram pressure strengthening of the

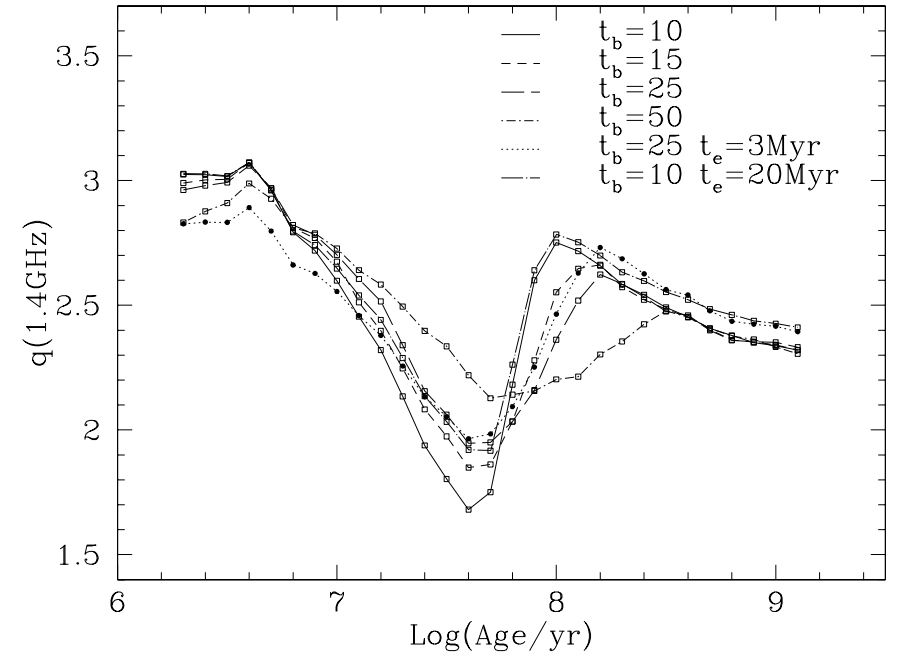

Fig. 8. The $q$ ratio as a function of time for the models in Fig. 7.

galaxy magnetic field, as the galaxy travels through the intracluster medium. According to Miller \& Owen (2001) this explanation seems not to work because of the lack of any correlation with the galaxy velocity. They suggest instead a compression of the galactic magnetic field by thermal pressure of the intracluster medium.

However, due to its small scatter, the FIR/radio correlation should be independent of the effects of the environment. We thus advance the hypothesis that the observed excess is due to an excess of post starburst galaxies in the central regions of the cluster. Indeed soon after a peaked starburst episode and/or a star formation interruption, the radio emission fades less rapidly than the FIR emission, causing an apparent radio enhancement. In this case the effect would be only indirectly due to the environment, as the gas rich galaxies enhance and/or exhaust their star formation through a central crossing.

All our models show a more or less pronounced minimum value of $q$ during the post starburst phase. The model with $t_{\mathrm{b}}=10 \mathrm{Myr}$ remains below $q_{1.49 \mathrm{GHz}}=2$ for about $20 \mathrm{Myr}$ (Fig. 8), while its luminosity at the minimum q has decreased by one order of magnitude (Fig. 6), but it is still infrared luminous. By decreasing the total mass formed in the burst it is easy to populate for a short time the region with $q_{1.49 \mathrm{GHz}}$ below 2 and $L_{\text {FIR }}$ between $10^{10}$ and $10^{11} L_{\odot}$. Thus a low value of $q_{1.4 \mathrm{GHz}}$ could simply be a natural consequence of the particular star formation history experienced by the galaxy. Because high resolution radio images probe that the emission is extended, the recent star formation history, either simply interrupted or enhanced and exhausted in a burst, must have been globally synchronised. Our models place an upper limit of less than $100 \mathrm{Myr}$ to the age of the last major burst and/or interruption of the star formation. At a typical velocity of $1000 \mathrm{~km} \mathrm{~s}^{-1}$, the galaxy has moved by only $100 \mathrm{kpc}$ since the beginning of the burst. This figure could be about two times larger if we allow for the formation of CCSN down to $5 M_{\odot}$ (overshoot models without $\mathrm{O}, \mathrm{Ne}, \mathrm{Mg}$ white dwarfs) and use a slightly slower star formation decline.

In summary, our models indicate that the star formation switch-off happened not too far from the present galaxy 
position. The excess of low $q$ galaxies is thus simply due to the larger probability of switching off the star formation in the higher density regions of the cluster. It is a nurture effect, possibly due to the higher degree of harassment suffered in the central regions. As a clear implication, our spectrophotometric models predict that these galaxies should show enhanced Balmer absorption features, e.g. EW $\mathrm{H} \delta \geq 5 \AA$ (see also Miller $\&$ Owen 2001). If our interpretation is correct, the analysis of the statistics of the deviation from the FIR/radio correlation at values of $q$ lower than the average, coupled with the low characteristic time involved, provides an independent measure of the local rate at which the Butcher-Oemler effect is operating within galaxy clusters.

\section{High redshift starbursts}

In the last few years, a wealth of observations performed in FIR/sub-mm spectral regions revealed the existence of a new class of galaxies interpreted as the high- $z$ analogue of the local ULIRGs (e.g. Smail et al. 1997; Hughes et al. 1998; Barger, Cowie, \& Sanders 1999). The sub-mm fluxes, probably mostly powered by star formation rather than AGN, (Granato et al. 1997; Almaini et al. 1999), imply star formation rates of several hundreds of $M_{\odot} / \mathrm{yr}$.

The discovery of these galaxies has introduced a new test of the theories of structure formation, but to this aim the knowledge of their redshift distribution is of fundamental importance. Due to the uncertain position, or to the lack, of the optical counterparts, a spectroscopic redshift is available only for a few sub-mm sources. Instead, an estimate of the redshift for most of these galaxies has been performed exploiting the FIR-radio correlation observed for local star forming galaxies, under the hypothesis that high- $z$ galaxies obey the same correlation as local ones (Carilli \& Yun 1999, 2000; Dunne et al. 2000; Yun \& Carilli 2002).

Due to the very different power laws of the sub$\mathrm{mm}$ and the radio spectra, the spectral index $s_{1.4}^{353} \equiv$ $\log \left(S_{353} / S_{1.4}\right) / \log (353 / 1.4)$, between the $850 \mu \mathrm{m}(353 \mathrm{GHz})$ and the $1.4 \mathrm{GHz}$ fluxes, depends very strongly on redshift, with the main uncertain factors in its effectiveness as a redshift indicator being (Carilli \& Yun 2000; Dunne et al. 2000): (a) the dust temperature distribution, (b) the slope of the dust emissivity in the sub-mm and (c) the slope of the radio spectrum. Additional uncertainties are (d) the assumed validity of the FIR-radio correlation at high- $z$, that recently has been directly tested to $z \sim 1.3$ (Garrett 2002), (e) the possible presence of AGNs (whose values of $s_{1.4}^{353}$ could be attributed to lower $z$ star forming galaxies rather than high- $z$ AGN), and (f) the quenching of the synchrotron photons due to inverse Compton of relativistic electrons off the CMB radiation, which is expected to be important for $z>6$ (Carilli \& Yun 1999).

With our model, we have investigated the $s_{1.4}^{353}(z)$ relation for starburst galaxies, taking into account the uncertainties $a, b$ and $c$. These factors affect the intrinsic shape of the galaxy SED. Uncertainties due also to observational and calibration errors are accounted for by Hughes et al. (2001). The $\mathrm{T}$ distribution of dust and its slope are degenerate in the sub-mm, because a shallower slope can mimic the effect of a

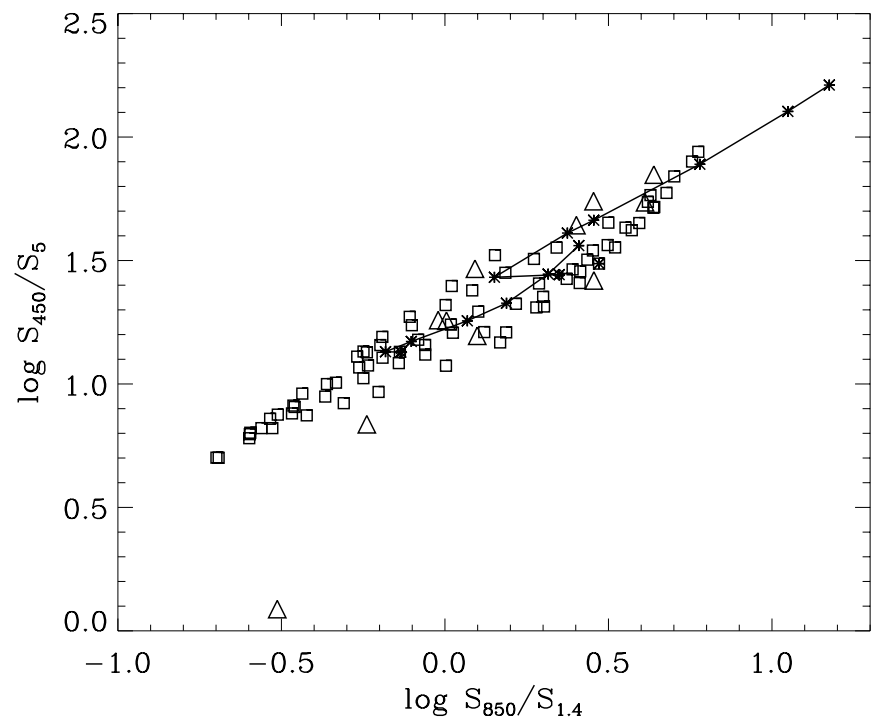

Fig. 9. Color-color plot $\log S_{850 \mu \mathrm{m}} / S_{1.4 \mathrm{GHz}}$ vs. $\log S_{450 \mu \mathrm{m}} / S_{5 \mathrm{GHz}}$. Squares: starburst models with $t_{\mathrm{b}}=5,10,15,25,50 \mathrm{Myr}$ evolved to an age of $3 t_{\mathrm{b}}$. Diamonds: models for M 82 and ARP 220 (see Figs. 1 and 2), reproducing the SED from the UV to the radio (see Silva et al. 1998 for details). Asteriscs connected by solid line: evolution during the starburst of the models reproducing M 82 and ARP 220. Triangles: data from Dunne et al. (2000), Condon et al. (1991).

colder dust component with a steeper slope (e.g. Silva 1999). This is due to the fact that the convolution of gray bodies of different temperatures (resulting from the $\mathrm{T}$ distribution of dust within a galaxy) yields a slope shallower than the effective one of the emissivity of dust. Moreover both the T distribution of dust in galaxies and the slope of the radio emission depend on several factors that change during the evolution of the starburst (i.e. the evolution of the stars heating the dust and their distribution across the galaxy, the dust optical depth, the relative importance of free-free and synchrotron emission and the amount of free-free absorption, see Sect. 5).

Therefore we have adopted the set of starburst models described in Sect. 5, i.e. the exponentially decreasing starbursts with e-folding times $t_{\mathrm{b}}=10,15,25,50 \mathrm{Myr}$, complemented with one more case, $t_{\mathrm{b}}=5 \mathrm{Myr}$, and with the models for M 82 and ARP 220 (Figs. 1 and 2), to study the dependence of $s_{1.4}^{353}(z)$ on the evolutionary status of the starbursts and the consequent different shapes of the SEDs. In particular, the model for ARP 220 needs a slope for the emissivity of dust in the sub-mm of 1.5, while this is not required for M 82 and other nearby galaxies (see Silva et al. 1998), whose SEDs can be reproduced with a slope of 2 . In Sect. 5 we showed that the starburst models cover the range of observed values of the FIR-radio relation and of the radio spectral index. In Fig. 9 we have checked that the range of values of the sub-mm to radio ratios $(850 \mu \mathrm{m}-1.4 \mathrm{GHz}$ and $450 \mu \mathrm{m}-5 \mathrm{GHz}$ to check also the spectral regions observed at high $z$ ) of the models, cover at least all the observed range. Furthermore, we can take into account also ratios not observed in the available local galaxy samples, but that might be expected to be present in some phases (e.g. during the first $\sim 3$ Myr since the onset of the starburst when the radio emission is mostly thermal). The models are 


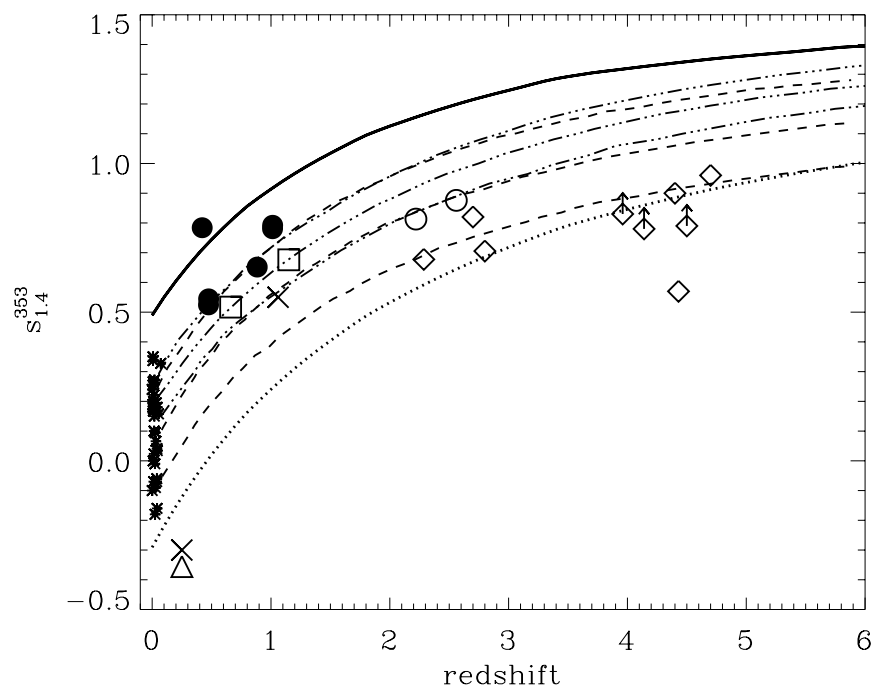

Fig. 10. The spectral index $s_{14}^{353}$ as a function of redshit for our models (upper continuos and lower dotted lines), and from Carilli \& Yun (2000) and Dunne et al. (2000) (respectively dashed and 3 dot-dashed lines for average and $\pm 1 \sigma$ ). The upper model curve corresponds to a very initial phase of the starburst, when free free radio emission dominates, while the lowest one is for a late phase. Data for galaxies and AGN with spectroscopic redshift are reported: asteriscs are local galaxies by Carilli \& Yun (2000) and Dunne et al. (2000); diamonds are AGN by Rowan-Robinson et al. (1993), Ivison et al. (1998), Yun et al. (2000); filled circles, crosses, squares, and circles are sub-mm and radio selected galaxies from Barger et al. (2000), Smail et al. (2000), Eales et al. (2000), and Ivison et al. (2000) respectively. The triangle is a cD galaxy from Edge et al. (1999).

evolved to an age of $3 t_{\mathrm{b}}$ since the start of the burst. In Fig. 10 we show the $s_{1.4}^{353}$ vs. $z$ relation resulting by considering all our models (we plot the highest and the lowest one, corresponding respectively to a very initial phase dominated by free free thermal radio emission and a late phase, dominated by NT radio emission), together with the relations by Carilli \& Yun (2000) and Dunne et al. (2000). As already remarked by these authors, the effect of low frequency free-free absorption on the relation is important only at very low $z$, due to its strong frequency dependence $\left(\tau_{v}^{\mathrm{ff}} \propto v^{-2.1}\right)$.

We note that the dispersion of local observations is entirely compatible with the one introduced by the evolution of the starburst. Furthermore, the different evolutionary stages and thus SED shapes of the models, result in a significant dispersion of $s_{1.4}^{353}$ at any $z$, sufficient to invalidate any quantitative estimate of $z$. We show that it might be possible to reduce the uncertainty in the redshift estimate by combining $s_{1.4}^{353}$ with another spectral index that depends mainly on the starburst age. The latter is a radio spectral index (see Sect. 5 and Fig. 7). Indeed for redshift between 0 and 6 the radio spectral index of each model changes less than 0.2. Thus one may use this index to confine the $s_{1.4}^{353}-z$ evolution within the possible values allowed by the corresponding selected starburst phase.

In Fig. 11 we show that a linear combination of the spectral index $s_{1.4}^{353}$ with a radio spectral index $(5-1.4 \mathrm{GHz}$ in the figures) may indeed reduce the redshift uncertainty at each $z$ originating from age dispersion. As an example, $\Delta z$ at $z=2$ for models is 3.12, 1.67, 0.82 respectively for Fig. 10 and
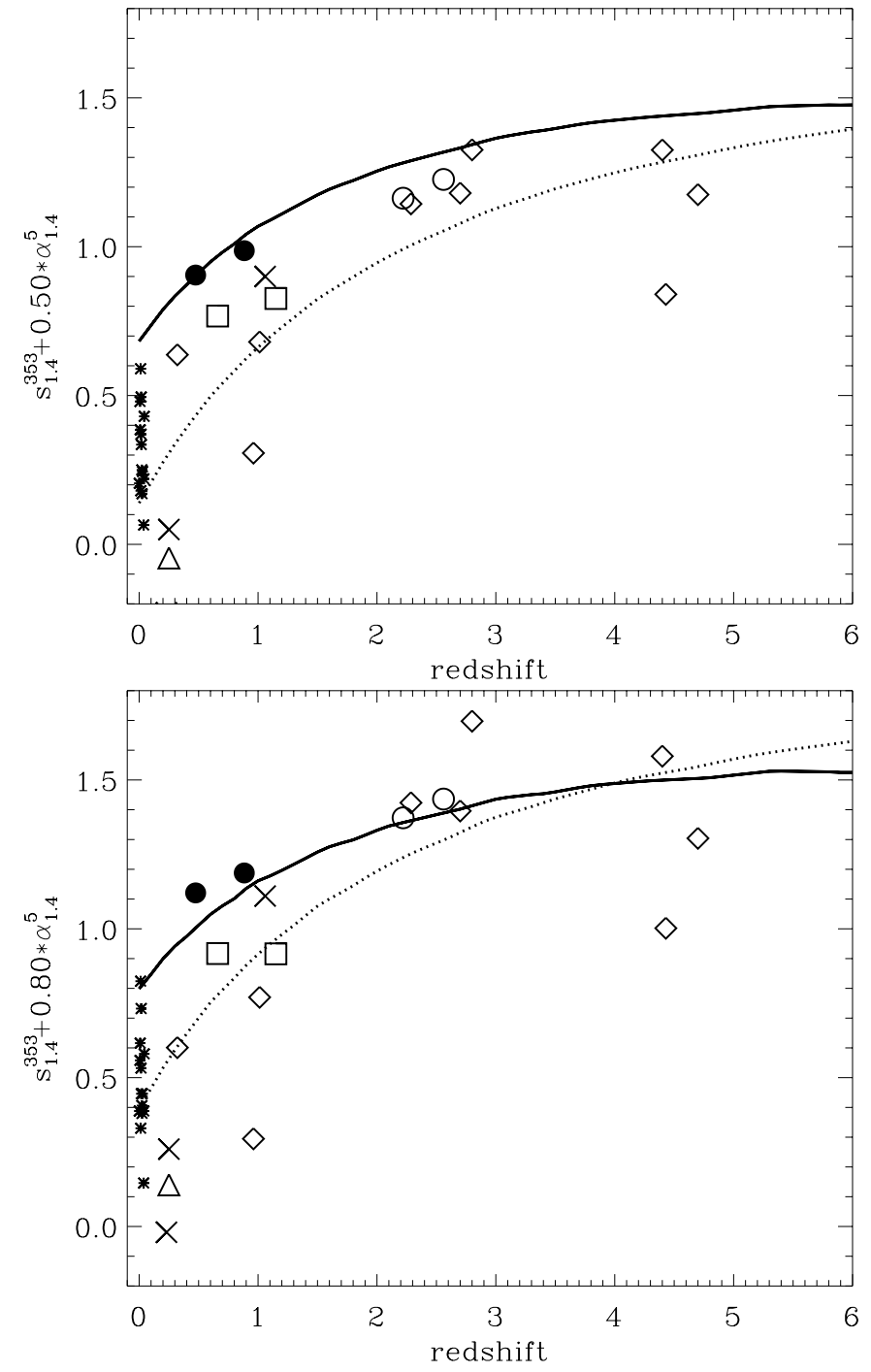

Fig. 11. Linear combinations of $s_{1.4}^{353}$ and $\alpha_{1.4}^{5}$ for models and data. For data with only one radio flux available, the radio spectral index was set to 0.7 . See Fig. 10 for references to data.

the two panels of Fig. 11. On the other hand it is important to note that, by considering the few available sources (excluding the known AGN) with a spectroscopic $z$, the redshift range that would correspond to their ordinate value depends on the adopted spectral index or combination. A detailed investigation of the redshift distribution of sub-mm galaxies is beyond the scope of this paper.

\section{Discussion and conclusions}

In this paper we have revisited the nature of the FIR/radio correlation observed in star forming galaxies. To understand its origin and range of validity we have utilized models of normal star forming and starburst galaxies. The infrared emission has been estimated with our population synthesis code, GRASIL, which is particularly suited for the prediction of the SED of star forming galaxies, from the UV to the sub-mm. For the radio emission we have adopted a model which extends the capabilities of GRASIL into the radio regime, essentially following 
the prescriptions given by Condon \& Yin (1990). Before adopting it we have reviewed all the possible sources of radio emission related to star formation activity, with particular emphasis on the integrated properties of stellar populations.

In agreement with previous studies, we have found that the fraction of the NT emission due to radio supernova remnants is about $6 \%$ of the total and that other discrete sources provide a negligible contribution. Almost all the NT emission thus come from diffuse electrons possibly injected into the interstellar medium by CCSN events, and adopting a relation between the NT emission and the CCSN rate seems the safest way to proceed. However, contrary to previous studies, we have also shown that the latter relation, which is at the base of the tightness of the FIR/radio correlation, is a natural outcome whenever synchrotron losses happen on timescales that are shorter than the fading time of the CCSN rate.

This is certainly the case in normal spirals, where the global SFR changed very little over the last billion years. But the situation may be very different in luminous starbursts, where the SN rate may change significantly on a timescale typical of the lifetime of the most massive stars. Indeed, previous studies claimed that the existence of a FIR/radio correlation under such conditions requires a fine tuning between the magnetic and radiative energy density, which is difficult to explain. We have shown that this fine tuning is not necessary. In fact the observed prevalence of synchrotron energy losses over inverse Compton losses indicates large magnetic fields and, consequently, guarantees very short synchrotron electron lifetimes (Condon 1992), certainly shorter than the typical lifetime of the most massive stars. The proportionality between the NT emission and the CCSN rate thus holds even in the extreme conditions found in the luminous obscured compact starbursts. This is why the FIR/radio correlation appears so robust.

We have calibrated the NT emission/CCSN rate relation on the observed properties of our Galaxy. With these assumptions we reproduce well the FIR/radio correlation of normal star forming galaxies, namely $q_{1.4 \mathrm{GHz}}=2.3$. We are thus quite confident that our model is able to reproduce both the FIR and Radio emission of star forming galaxies, with a minimum set of well-calibrated parameters.

As for the starburst galaxies, with a rapidly changing SFR, we have shown that the different fading times of the FIR and Radio emission may be used to analyse in great detail the recent star formation history in these galaxies. Indeed, in the cases of M 82 and ARP 220, presented in Sect. 5, the combination of FIR and Radio observations allows us to reach a time resolution of a few tens of Myr, which is not possible based on the UV-FIR continuum properties alone, and even on the optical/NIR emission line diagnostics for obscured galaxies (Poggianti et al. 2001; Bressan et al. 2001). We have thus analysed in greater detail the evolution of obscured starburst galaxies under different assumptions concerning the burst characteristics, challenged by the possibility of using the deviations from the FIR/radio correlation as a diagnostic tool to infer the recent star formation history. We have compared our results with observations of a sample of compact ULIRGs, having in mind that, in these objects, it is also not clear what fraction of IR and radio emission is possibly contributed by the AGN.
Compact ULIRGs show a noticeable deviation from the average FIR/radio relation, with their $q_{1.49 \mathrm{GHz}}$ being generally lager than 2.3 , a few of them with values as high as 3 . Taken at face value, these deviations suggest that radio emission is depressed by a large factor relative to normal spirals. Understanding the interplay between FIR and radio emission in these objects is thus fundamental to make reliable predictions for high redshift dust-enshrouded galaxies.

Starburst models with peak SFR reaching several hundred $M_{\odot} / \mathrm{yr}$ and thereafter exponentially declining may account for the IR and radio emission of the observed ULIRGs and are able to reproduce the observed variation of the value of $q_{1.49 \mathrm{GHz}}$. This view is consistent with the current idea that ULIRGs and, to a larger extent high redshift dust enshrouded galaxies, are transient phenomena that nevertheless build up a significant fraction of stars and metals (Granato et al. 2001).

The introduction of a new diagnostic diagram, the $q_{1.49 \mathrm{GHz}}$ vs. radio slope diagram, allows us to single out the effects of starburst evolution and free-free absorption. Very young star bursts display an excess of FIR emission relative to the radio emission because the latter is initially contributed mainly by the free-free emission process. As the starburst ages, the NT contribution increases and becomes the dominant source, while the radio slope reaches the typical values observed in synchrotron emission. Free-free absorption affects the $1.49 \mathrm{GHz}$ data, introducing a trend with a higher $q$ being accompanied by a shallower slope. The estimated optical depths for free-free absorption at $1.49 \mathrm{GHz}$ are between 0.5 and 1 . At $8.4 \mathrm{GHz}$, free-free absorption becomes negligible and the above trend disappears. The value of $q_{8.4 \mathrm{GHz}}$ is a measure of the age of the starburst. However, even in the latter diagram the slope is still affected by free-free absorption. Thus we suggest that a similar diagram between $8.4 \mathrm{GHz}$ and a higher frequency range would be critical for the understanding of the evolutionary status of compact ULIRGs because, in that case, the slope, unaffected by free-free absorption, would provide an independent estimate of the age.

If ULIRGs are transient phenomena as suggested by other independent studies, then determining their SFR from conventional estimators may be a problem. They are far from being stationary; the term "average star formation" is meaningless, and applying standard calibrations may result in a significant error and/or apparent discrepancy between the observations themselves. One should be able to reconstruct the recent history of star formation and, for this purpose, we suggest the use of the above diagram to determine the characteristic parameters of the burst first, and then the age-averaged SFR from either the FIR or radio luminosity.

Another relevant question addressed is how reliable the use the FIR/radio correlation is to evaluate the contribution of nonthermal radiation from the central active nucleus. In the plotted data, the symbol "M" indicates the position of the Seyfert 1 galaxy Mrk 231 (UGC 08058) (Thean et al. 2000). The fact that Mrk 231 is clearly distinct from the other objects and occupies a position below any starburst model becomes particularly evident in the $q_{8.4 \mathrm{GHz}}$ vs. slope diagram, where the effects of free-free absorption on the $q$ ratio are minimized.

We have also shown that during the post starburst phase, the models reach values of $q$ significantly lower than those 
of quiescent spirals, with still significant FIR luminosities. This is consistent with the detection in nearby Abell clusters of a statistically significant excess of star forming galaxies with enhanced radio emission relative to the FIR (Miller \& Owen 2001). We suggest that these low values of $q$ are due to an evolutionary effect rather than a direct enhancement of radio emission by interaction with the intracluster medium.

Finally we have investigated the redshift dependence of the FIR/radio correlation and its validity (through the sub-mm radio index, $s_{1.4}^{353}$ ) to provide a photometric redshift estimate of obscured distant galaxies. The large dispersion of $s_{1.4}^{353}$ observed among local galaxies is compatible with the evolutionary effects discussed in Sect. 5. The unknown evolutionary status of the starburst renders the $s_{1.4}^{353}$ index very unreliable at almost any redshift. We thus suggest complementing the in$\operatorname{dex} s_{1.4}^{353}$ with a radio slope determination, because of its tight relation with the evolutionary phase of the starburst and the its very shallow dependence on the redshift. Other uncertainties like the sub-mm slope and the presence of a significant contribution at radio wavelengths from a central AGN obviously worsen the above picture.

Acknowledgements. We thank I. Aretxaga, L. Danese, A. Franceschini, D. Huges, P. Panuzzo, B. Poggianti and O. Prouton for useful discussions and the anonymous referee for useful comments. A.B. and G.L.G. acknowledge warm hospitality by INAOE. This research was partially supported by the European Community under TMR grant ERBFMRX-CT96-0086 and by the Italian Ministry for University and Research (MURST) under grant Cofin 92001021149-002.

\section{References}

Almaini, O., Lawrence, A., \& Boyle, B. J. 1999, MNRAS, 305, L59 Barger, A. J., Cowie, L. L., \& Sanders, D. B. 1999, ApJ, 518, L5 Barger, A. J., Cowie, L. L., \& Richards, E. A. 2000, AJ, 119, 2092 Berkhuijsen, E. M. 1984, A\&A, 140, 431

Berta, S., Fritz, J., Pernechele, C., Bressan, A., \& Franceschini, A. 2002, ApJ, submitted

Blain, A. W. 1999, MNRAS, 309, 955

Bressan, A., Poggianti, B., \& Franceschini, A. 2001, QSO hosts and their environments (Dordrecht: Kluwer Academic/Plenum Publishers), xvii, 376, ISBN 0306466627, 171

Calzetti, D., Kinney, A. L., \& Storchi-Bergmann, T. 1994, ApJ, 429, 582

Carilli, C. L., \& Yun, M. S. 1999, ApJ, 513, L13

Carilli, C. L., \& Yun, M. S. 2000, ApJ, 530, 618

Carilli, C. L. 2000, Radio Observations of High Redshift Star Forming Galaxies, in Starburst Galaxies Near and Far, ed. D. Lutz, \& L. Taconni (Springer) [astro-ph/0011199]

Clark, D. H., \& Caswell, J. L. 1976, MNRAS, 174, 267

Condon, J. J. 1992, ARA\&A, 30, 575

Condon, J. J., \& Yin, Q. F. 1990, ApJ, 357, 97

Condon, J. J., Huang, Z. P., Yin, Q. F., \& Thuan, T. X. 1991, ApJ, 378, 65

Dunne, L., Clements, D. L., \& Eales, S. A. 2000, MNRAS, 319, 813

Dwek, E., Arendt, R. G., Hauser, M. G., et al. 1998, ApJ, 508, 106

Eales, S., Lilly, S., Webb, T., et al. 2000, AJ, 120, 2244

Edge, A. C., Ivison, R. J., Smail, I., Blain, A. W., \& Kneib, J. P. 1999, MNRAS, 306, 599

Elbaz, D., Cesarsky, C. J., Fadda, D., et al. 1999, A\&A, 351, L37
Ferland, G. J. 1996, Hazy, a Brief Introduction to CLOUDY 90, Univ. of Kentucky Physics Department Internal Report

Flores, H., Hammer, F., Thuan, T. X., et al. 1999, ApJ, 517, 148

Fritz, J., Berta, S., Pernechele, C., Bressan, A., \& Franceschini, A. 2002, ApJ, submitted

Garrett, M. A. 2002, A\&A, 384, L19

Goldader, J. D., Meurer, G., Heckman, T. M., et al. 2002, ApJ, 568, 651

Gavazzi, G., \& Jaffe, W. 1986, ApJ, 310, 53

Gordon, S. M., Duric, N., Kirshner, R. P., Goss, W. M., \& Viallefond, F. 1999, ApJS, 120, 247

Granato, G. L., Danese, L., \& Franceschini, A. 1997, ApJ, 486, 147

Granato, G. L., Lacey, C. G., Silva, L., et al. 2000, ApJ, 542, 710

Granato, G. L., Silva, L., Monaco, P., et al. 2001, MNRAS, 324, 757

Hauser, M. G., Arendt, R. G., Kelsall, T., Dwek, E., et al. 1998, ApJ, 508, 25

Helou, G., Khan, I. R., Malek, L., \& Boehmer, L. 1988, ApJS, 68, 151

Hughes, D. H., et al. 1998, Nature, 394, 241

Hughes, D. H., et al. 2001 [astro-ph/0111547]

Ivison, R. J., Smail, I., Le Borgne, J. F., et al. 1998, MNRAS, 298, 583

Ivison, R. J., Smail, I., Barger, A. J., et al. 2000, MNRAS, 315, 209

Kafatos, M., Sofia, S., Gull, T., \& Bruhweiler, F. 1980, ApJ, 242, 294

Lisenfeld, U., Voelk, H. J., \& Xu, C. 1996, A\&A, 314, 745

Longair, M. S. 1994, High energy astrophysics, vol. 2: Stars, the galaxy and the interstellar medium (Cambridge University Press), 280

Meurer, G. R., Heckman, T. M., \& Calzetti, D. 1999, ApJ, 521, 64

Miller, N. A., \& Owen, F. N. 2001, AJ, 121, 1903

Panuzzo, P., Granato, G. L., Bressan, A., Silva, L., \& Danese, L. 2002, A\&A, submitted

Pernechele, C., Bonoli, C., Bressan, A, et al. 2002, ApJ, submitted

Poggianti, B. M., \& Wu, H. 2000, ApJ, 529, 157

Poggianti, B. M., Bressan, A., \& Franceschini, A. 2001, ApJ, 550, 195

Portinari, L., Chiosi, C., \& Bressan, A. 1998, A\&A, 334, 505

Puget, J.-L., Abergel, A., Bernard, J.-P., et al. 1996, A\&A, 308, L5

Renzini, A., \& Buzzoni, A. 1986, in Spectral evolution of galaxies, ed. C. Chiosi, \& A. Renzini (Reidel, Dordrecht), 195

Ritossa, C., Garcia-Berro, E., \& Iben, I. Jr. 1996, ApJ, 460, 489

Rowan-Robinson, M., Efstathiou, A., Lawrence, A., et al. 1993, MNRAS, 261, 513

Rowan-Robinson, M. 1995, MNRAS, 272, 737

Rubin, R. H. 1968, ApJ, 154, 391

Sanders, D. B., \& Mirabel, I. F. 1996, ARA\&A, 34, 749

Silva, L., Granato, G. L., Bressan, A., \& Danese, L. 1998, ApJ, 509, 103

Silva, L. 1999, Ph.D. Thesis, SISSA/ISAS, Trieste, Italy

Smail, I., Ivison, R. J., \& Blain, A. W. 1997, ApJ, 490, L5

Smail, I., Ivison, R. J., Owen, F. N., Blain, A. W., \& Kneib, J. P. 2000 , ApJ, 528, 612

Smith, H. E., Lonsdale, C. J., \& Lonsdale, C. J. 1998, ApJ, 492, 137 (SLL)

Soifer, B. T., Sanders, D. B., Neugebauer, G., et al. 1986, ApJ, 303, L41

Soifer, B. T., et al. 2000, AJ, 119, 509

Thean, A., Pedlar, A., Kukula, M. J., Baum, S. A., \& OD́ea, C. P. 2000, MNRAS, 314, 573

Veilleux, S., Kim, D.-C., Sanders, D. B., Mazzarella, J. M., \& Soifer, B. T. 1995, ApJS, 98, 171

Valdes Parra, J. R., Rodighiero, J., Bressan, A., Franceschini, A., \& Rigopoulou, D. 2002, in preparation

Wu, H., Zou, Z. L., Xia, X. Y., \& Deng, Z. G. 1998b, A\&AS, 132, 181

Yun, M. S., Carilli, C. L., Kawabe, R., et al. 2000, ApJ, 528, 171

Yun, M. S., \& Carilli, C. L. 2002, ApJ, 568, 88 